\title{
Online friction parameter estimation for machine tools
}

\section{Papageorgiou, Dimitrios; Blanke, Mogens; Niemann, Hans Henrik; Richter, Jan H.}

Published in:

Advanced Control for Applications: Engineering and Industrial Systems

Link to article, DOI:

10.1002/adc2.28

Publication date:

2020

Document Version

Peer reviewed version

Link back to DTU Orbit

Citation (APA):

Papageorgiou, D., Blanke, M., Niemann, H. H., \& Richter, J. H. (2020). Online friction parameter estimation for machine tools. Advanced Control for Applications: Engineering and Industrial Systems, [e28].

https://doi.org/10.1002/adc2.28

\section{General rights}

Copyright and moral rights for the publications made accessible in the public portal are retained by the authors and/or other copyright owners and it is a condition of accessing publications that users recognise and abide by the legal requirements associated with these rights.

- Users may download and print one copy of any publication from the public portal for the purpose of private study or research.

- You may not further distribute the material or use it for any profit-making activity or commercial gain

- You may freely distribute the URL identifying the publication in the public portal

If you believe that this document breaches copyright please contact us providing details, and we will remove access to the work immediately and investigate your claim. 
DOI: $10.1002 / \mathrm{adc} 2.28$

\title{
ORIGINAL ARTICLE
}

\section{Online Friction Parameter Estimation for Machine Tools}

\author{
Dimitrios Papageorgiou*1 | Mogens Blanke ${ }^{1}$ | Hans Henrik Niemann ${ }^{1}$ | Jan H. Richter ${ }^{2}$
}

${ }^{1}$ Department of Electrical Engineering, Technical University of Denmark, Elektrovej 326, 2800 Kgs. Lyngby, Denmark, Email: dimpa,mb,hhn@elektro.dtu.dk ${ }^{2}$ Digital Factory Division, Siemens AG, D-90475 Nuremberg, Germany, Email: janrichter@siemens.com

\section{Correspondence}

*Dimitrios Papageorgiou. Email:

dimpa@elektro.dtu.dk

\begin{abstract}
Summary
Accurate description of frictional phenomena is essential in applications that require high-accuracy control. Online monitoring of friction parameters in machine tools greatly improves the control accuracy making condition-based feedforward compensation possible and, at the same time, facilitates equipment wear assessment, which enables efficient scheduling of maintenance. Existing friction estimation methods that use detailed dynamical models offer accurate description of friction phenomena, but often rely on a-priori knowledge of the static friction parameters, which have to be identified offline. This paper suggests an adaptive estimation strategy suitable for online use while the machine works in its normal production cycle. Smooth approximations to are introduced to account for stiction, viscous and bidirectional Coulomb friction in order to make online estimation possible. A parallel architecture is used with two adaptive estimators that segregate the frictional phenomena that dominate in different parts of the motion regime. Stability properties are analysed and performance is experimentally validated on a single-axis state-of-the-art industrial test rig.
\end{abstract}

\section{KEYWORDS:}

Friction estimation, machine tool drive, stiction, adaptive estimator, nonlinear parameterisation, experimental validation, wear assessment, cascaded systems.

\section{1 | INTRODUCTION}

Friction in electromechanical systems is a very complex phenomenon with many nonlinear aspects. In machine tools applications, where high accuracy position and velocity control is required, an accurate description of friction is essential. Friction in machine tool drive-trains primarily develops between the contacting bearings of the drive motor, as well as between the surfaces of the linear axes. Gearbox lubricants introduce viscous friction, while heat losses in the drive (e.g. due to eddy currents), may change viscosity under operation. Static friction (stiction) significantly contributes to the degrading of the linear axes positioning ${ }^{1,2}$, especially during motion onsets and axes reversals ${ }^{3}$. Sufficient knowledge of the occurring frictional phenomena, not only facilitates better control loops but may also infer information about the levels of equipment wear. For example increased dry friction can be associated to wear in the axis ball screws or the slides' surfaces, while increased viscosity may imply lubrication film failure ${ }^{4}$. Stiction, Coulomb- and viscous friction constitute the main friction elements of concern and estimation of their characteristic parameters is highly desirable for both control and maintenance.

A large number of models, both static and dynamic, have been proposed in the literature for effectively describe friction. A detailed survey of these models was presented in ${ }^{5}$ and more recently in ${ }^{6,7,8}$, where the Dahl, LuGre and the Generalised Maxwell-Slip (GMS) friction models were discussed in detail. These models describe friction based on the dynamic behaviour 
of the bristles of the various contacting surfaces in a mechanical system and include nonlinear phenomena, such as presliding (Stribeck effect) and hysteresis. The Lueven model, presented in ${ }^{9}$ and ${ }^{10}$, was developed based on modifying the dynamical state equation of the LuGre model for accurate hysteresis description. Additional single-state and multi-state dynamical models have been developed as combinations or extensions of the previous ones. Specifically, the authors in ${ }^{11}$ proposed a single-state integral model of friction as an extension of the Dahl model by including a term for micro-damping. A multi-state description is pursued in ${ }^{12}$, where each of the states represents the position of a single microscopic elastoslide element. Hysteresis and presliding nonlinear behaviour was successfully described by a two-state elastoplastic model in ${ }^{13}$, where the proposed description was an extension of the LuGre model. A simple dynamical friction model based on a smoothened version of the LuGre description was discussed in ${ }^{14}$. Time-series oriented friction models were presented in ${ }^{15}$. Such models provide a very effective framework for identification but they lack the direct connection to the actual physical parametrization of the different aspects of the nonlinear friction phenomena.

Estimation of friction and identification of different friction models has also been extensively addressed. Off-line optimization was used in ${ }^{16}$ for identification of the LuGre parameters. Different parts of the velocity-friction map were utilized for independent estimation of the parameters of the static and the dynamic parts of the model. Identification of LuGre friction in a rotating robotic arm system was performed in ${ }^{17}$. They integrated friction dynamics into a system model and used frequency responses of a linearized augmented system for friction parameter estimation through a series of dedicated experiments, each for different motion regimes. A similar separation strategy was followed in ${ }^{18}$, who employed least squares regression, graphical curve fitting and nonlinear optimization to identifiy the Karnopp friction model. Linearization of a combined friction-plant model was also used in ${ }^{19}$, who made offline friction parameter estimation by polynomial curve fitting and least-squares regression. The authors in $^{20}$ followed the same system-friction augmented description to compare different LuGre friction estimation algorithms based on the Extended Kalman Filter (EKF), Unscented Kalman Filter (UKF), Particle Filter (PF) and Differential Evolution (DE) method. A different approach was made in ${ }^{21}$, where the nonlinear friction relay behaviour was modelled with describing functions. The output limit cycles parameters, which give an implicit description of friction, were identified via offline nonlinear least squares optimization. The authors in ${ }^{22}$ also approached the estimation problem in frequency domain. Specifically, identification of the Dahl friction resonance characteristics was carried out, which reflects the frequency response of the limit cycle behaviour of friction in the presliding regime. Particle swarm optimization was used in $^{23}$ for LuGre friction estimation. The static and dynamic parameters were again estimated separately. The combination of three different offline optimization-based methods for friction identification was demonstrated in ${ }^{24}$. The estimation scheme utilized friction regime-specific properties based on LuGre and Maxwell-Slip models. Dynamical friction estimation was demonstrated in ${ }^{25}$, where identification was done using recursive least squares on a discrete time series approximation of the friction dynamics.

The majority of the reported work assumes dedicated experiments corresponding to the identification of separate friction components, which are usually identified offline. This is more frequent when models with several parameters are used, and even more when these models include friction dynamics, in which case the static parameters have to be available in advance. Linearization-based methods are easy to implement but the validity of the results can be limited to a narrow region around the operation point. A method for online identification of the LuGre friction model was demonstrated in ${ }^{26}$. A restriction of this approach is the requirement for a closed-loop scheme with feed-forward friction compensation. Online estimation techniques based on time-series models ${ }^{24,27}$ may provide accurate description of friction, but the relevant parametrization often departs from the physical meaning of the true friction parameters. Such approaches may compromise online monitoring of the friction parameters that is intended for wear assessment.

This paper proposes a new smooth static model for friction in machine tool systems that captures the phenomena of importance to high accuracy control and it presents a modular scheme for online estimation of the model parameters. Similar to the classic Coulomb/viscous friction and Stribeck models ${ }^{28}$, the proposed representation employs a first-principle parametrization that associates the model coefficients to the real aspects of friction, while it explicitly accounts for stiction and does not contain any discontinuities. In comparison to smooth models like the ones described in ${ }^{29}$, it maintains low complexity since it does not include the dynamics of the bristles. Specifically, a single-parameter smooth model for static friction is proposed, while a modified smooth version of the Coulomb-viscous friction model with three unknown parameters is introduced to account for different Coulomb constants for positive and negative velocities. The two models are combined in a unified friction description based on a motion-regime separation function. The proposed model aims to accurately describing the quasi-discontinuous characteristics of the friction processes, while maintaining sufficient levels of smoothness that is necessary for numerically-robust online estimation. The model is used in a cascaded scheme comprising a velocity observer and two adaptive estimators for online friction parameters estimation. Test rig experiments show the efficacy of the method. 


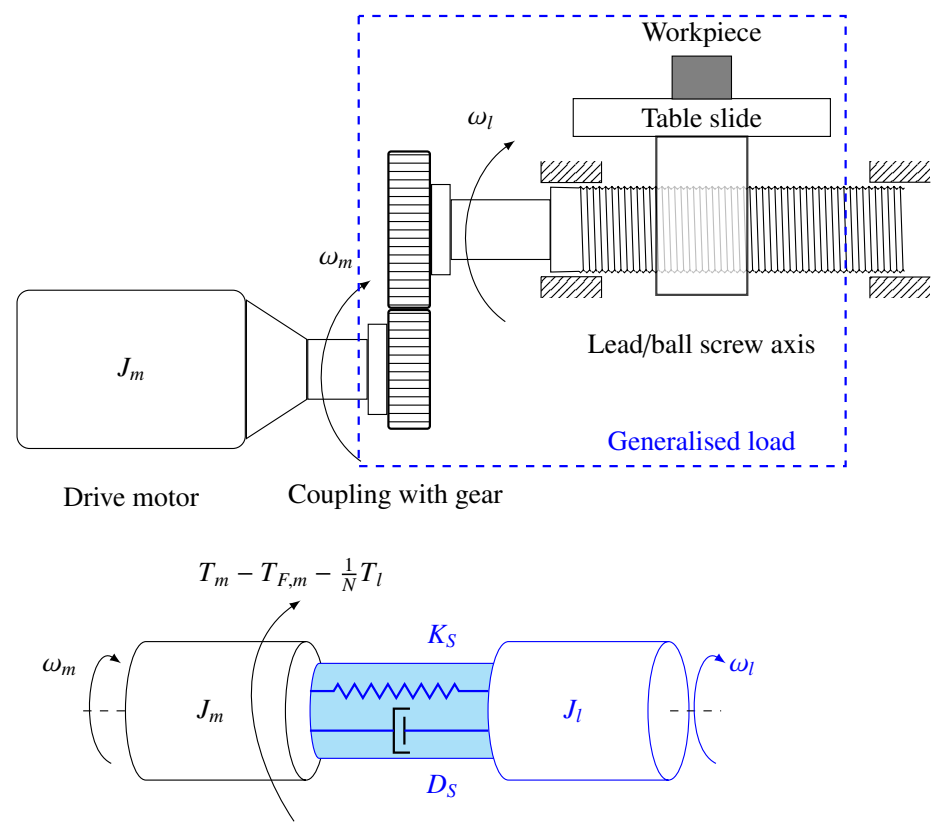

FIGURE 1 Correspondence between mechanical drive-train and machine-tool axis, where $\omega_{m}, \omega_{l}$ are the motor and load angular velocities, respectively. In the bottom plot, $J_{m}, J_{l}$ are the drive motor and generalised load inertias, respectively, $T_{m}$ is the torque generated by the drive motor, $T_{l}$ is the interconnecting spring-damping torque, $K_{S}, D_{S}$ are the spring constant and damping coefficient of the flexible shaft, respectively and $T_{F, m}$ is the friction torque acting on the motor.

The paper is organized as follows: Section 2 states the estimation problem discussed in this study, describes the drive-train system and presents the modified friction model. Section 3 analytically describes the design of the friction estimation scheme based on the motion regime separation and provides the relevant convergence and stability proofs. Experimental results are presented in Section 4 to validate the theoretical findings, while Section 5 discusses various implementation aspects of the estimation scheme, especially with respect to robustness. Finally, conclusions are drawn and future work is outlined in Section 6.

\section{2 | SYSTEM MODELLING AND PROBLEM FORMULATION}

\section{1 | Drive-train modelling}

A machine tool axis consists of a feed drive, typically an AC motor with a power converter, and a linear axis (lead/ball screw) that connects to the motor shaft through a gearing system. The linear axis is often modelled as the interconnection of several connected mass-spring-damper systems ${ }^{3}$. These masses can be lumped into a single inertia and the combined elasticity and damping of the torsional springs allow the axis to be represented as a generalised load, which can be extended to include the machining tool, the workpiece etc. The entire machine axis can finally be described as a drive-train that consists of the drive motor and the generalised load, connected to each other via a flexible shaft with damping. This abstraction of the machine axis as a two-mass oscillator (motor-load) is illustrated in Figure 1.

The dynamics of the drive-train reads:

$$
\begin{aligned}
J_{m} \dot{\omega}_{m} & =u-T_{F, m}\left(\omega_{m}\right)-\frac{1}{N} T_{l}(\boldsymbol{x}) \\
\dot{\theta}_{m} & =\omega_{m} \\
J_{l} \dot{\omega}_{l} & =-T_{F, l}\left(\omega_{l}\right)+T_{l}(\boldsymbol{x}) \\
\dot{\theta}_{l} & =\omega_{l}
\end{aligned}
$$

where $x=\left[\omega_{m} \theta_{m} \omega_{l} \theta_{l}\right]^{T}$ is the system state vector containing angular velocities and positions for the motor and the load, respectively, $u$ is the torque generated by the drive motor, $J_{m}, J_{l}$ are the motor and load inertias, respectively, $N$ is the gearing 
ratio, $T_{F, m}, T_{F, l}$ are the total friction torques on the motor and the load, and $T_{l}$ is the interconnecting torque between motor and load. If there are no clearances between motor and load, $T_{l}$ is given from:

$$
T_{l}(\boldsymbol{x})=K_{S}\left(\frac{1}{N} \theta_{m}-\theta_{l}\right)+D_{S}\left(\frac{1}{N} \omega_{m}-\omega_{l}\right) .
$$

where $K_{S}, D_{S}$ are the shaft stiffness and damping coefficient, respectively, assumed known and constant in this study, i.e.

Assumption 1. The shaft coefficients $K_{S}, D_{S}$ and the motor inertia $J_{m}$ are constant and known from offline identification.

In the following the total torque opposing the motor friction will be referred to as the actuating torque and it is defined as:

$$
u_{a} \triangleq u-\frac{1}{N} T_{l}(x) \text {. }
$$

\section{2 | Friction modelling}

The friction torque $T_{F, m}$ acting on the motor is described by the following equation:

$$
T_{F, m}\left(\omega_{m}, u_{a}\right)=\left[1-\operatorname{sgn}^{2}\left(\omega_{m}\right)\right] T_{F, S}\left(u_{a}\right)+\left[\operatorname{sgn}\left(\omega_{m}\right) T_{C}+\beta \omega_{m}\right]
$$

where the first term is the static friction and the second term is the well-known Coulomb and viscous friction ${ }^{30}$, with $T_{C}$ being the Coulomb friction constant and $\beta$ the viscous friction coefficient. The expression $T_{F, S}$ denotes the static friction function, a theoretical expression of which is given by 31,32

$$
T_{F, S}\left(u_{a}\right)= \begin{cases}u_{a} & ,\left|u_{a}\right| \leq T_{S} \\ 0 & ,\left|u_{a}\right|>T_{S},\end{cases}
$$

or equivalently

$$
T_{F, S}\left(u_{a}\right)=\frac{u_{a}}{2}\left[1-\operatorname{sgn}\left(\left|u_{a}\right|-T_{S}\right)\right]
$$

where $T_{S}$ is the static friction constant. This definition of stiction implies that while the input torque is smaller than the static friction constant, the stiction develops such that it completely cancels the actuating torque $u_{a}$, preventing the motor from moving. When the motor generates enough torque to overcome $T_{S}$, then motion begins, the static friction vanishes and the friction torque contains only its Coulomb and viscous part. To avoid the two-point discontinuity in Equation (2.8), the static friction function during zero velocity can be smoothly parametrized with respect to $u_{a}, T_{S}$ as:

$$
T_{F, S}\left(u_{a}, T_{S}\right) \triangleq \frac{u_{a}}{\pi}\left[\tan ^{-1}\left(\alpha\left(u_{a}+T_{S}\right)\right)-\tan ^{-1}\left(\alpha\left(u_{a}-T_{S}\right)\right)\right]
$$

where $\alpha$ is a positive real number that defines the slope of the stiction function at the points of discontinuity. Higher values of $\alpha$ correspond to steeper models and for $\alpha \rightarrow \infty$, the approximated stiction function converges to the discontinuous one in (2.8) as it can be seen in Figure 2.

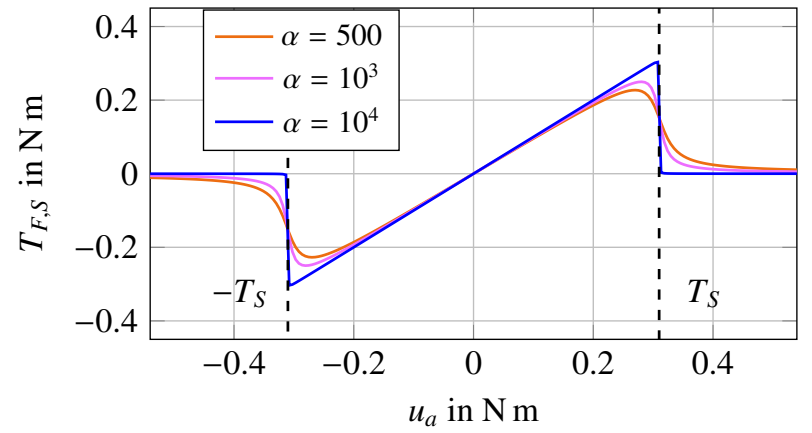

FIGURE 2 Static fiction during the "zero-velocity" regime as a function of the motor accelerating torque. Higher values of $\alpha$ correspond to steeper transitions into and out of the zone $\pm T_{S}= \pm 0.31 \mathrm{~N} \mathrm{~m}$. 
With the signum function conventionally defined ${ }^{1}$ as

$$
\operatorname{sgn}(y) \triangleq \begin{cases}1 & , y>0 \\ 0 & , y=0 \\ -1 & , y<0,\end{cases}
$$

it is clear from Equation (2.7) that when $\omega_{m}=0$, only $T_{F, S}$ contributes to total frictional torque, while for non-zero velocities, $T_{F, m}=T_{C} \operatorname{sgn}\left(\omega_{m}\right)+\beta \omega_{m}$. It is, therefore, convenient for estimation purposes to separate the motion of the rotor shaft in two regimes, i.e. one for $\omega_{m}=0$ and one for $\omega_{m} \neq 0$ as illustrated in Figure 3 .

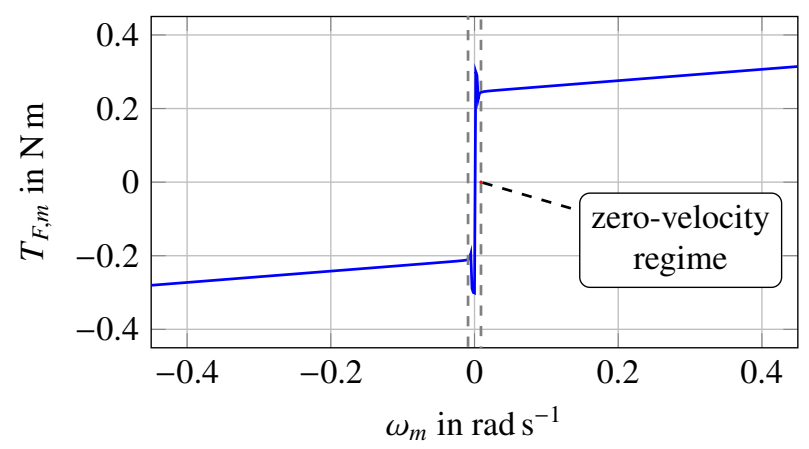

FIGURE 3 Motor friction as a function of the angular velocity. When $\omega_{m}=0$, the friction is equal to the stiction $T_{F, S}$. For non-zero velocities Coulomb and viscous friction constitute the total frictional torque. In general, the static friction coefficient is larger in magnitude than the Coulomb friction which is illustrated by the small peaks close to zero velocity. Here, $T_{S}=$ $0.31 \mathrm{~N} \mathrm{~m}, T_{C}^{+}=0.245 \mathrm{~N} \mathrm{~m}, T_{C}^{-}=0.211 \mathrm{~N} \mathrm{~m}, \beta=0.154 \mathrm{~N} \mathrm{~s}$.

In the presence of sensor noise it is difficult to accurately determine when the motor angular velocity is zero. For this reason, it is more useful to replace the term $\operatorname{sgn}\left(\omega_{m}\right)$ with a smooth function of $\omega_{m}$ that also includes a small deadzone around $\omega_{m}=0$. The width of the deadzone can be selected according to the noise levels of the velocity measurements. The signum function is, therefore, modified to

$$
s_{d}\left(\omega_{m}\right) \triangleq \frac{1}{\pi}\left[\tan ^{-1}\left(p\left(\omega_{m}-\omega_{t h}\right)\right)+\tan ^{-1}\left(p\left(\omega_{m}+\omega_{t h}\right)\right)\right]
$$

where $2 \omega_{t h}$ is the velocity deadzone width and $p$ is a positive scalar that defines the steepness of the modified signum function. The larger the value of $p$, the steeper the function. Figure 4 shows a plot of the modified signum function against the motor angular velocity $\omega_{m}$.

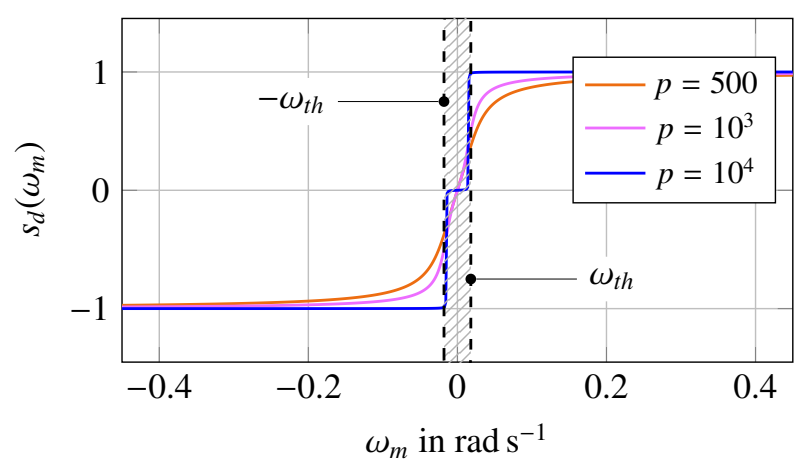

FIGURE 4 Modified signum function that includes a deadzone of width equal to $2 \omega_{t h}$, with $\omega_{t h}=0.015 \mathrm{~m} / \mathrm{sec}$.

\footnotetext{
${ }^{1}$ According to the more strict mathematical definition, $\operatorname{sgn}(0) \in[0,1]$.
} 
Another assumption often taken in online friction estimation methods using static models is that the Coulomb friction constant is the same for all nonzero velocity values. In reality the magnitude of Coulomb friction varies between two different constant values $T_{C}^{+}$and $T_{C}^{-}$, one for positive and one for negative angular velocities, respectively. The following model of the Coulomb friction magnitude, proposed in this study, facilitates a smooth transition from $T_{C}^{-}$to $T_{C}^{+}$, including at the same time the zerovelocity deadzone introduced earlier for increased robustness with respect to sensor noise:

$$
T_{C}\left(\omega_{m}, T_{C}^{+}, T_{C}^{-}\right) \triangleq \frac{1}{\pi}\left[\frac{\pi}{2}\left(T_{C}^{+}+T_{C}^{-}\right)+T_{C}^{+} \tan ^{-1}\left(\alpha\left(\omega_{m}-\omega_{t h}\right)\right)-T_{C}^{-} \tan ^{-1}\left(\alpha\left(\omega_{m}+\omega_{t h}\right)\right)\right]
$$

The complete, smooth friction model parametrization is given by:

$$
T_{F, m}\left(\omega_{m}, u_{a}, T_{S}, T_{C}^{+}, T_{C}^{-}, \beta\right)=\underbrace{\left[1-s_{d}^{2}\left(\omega_{m}\right)\right] T_{F, S}\left(u_{a}, T_{S}\right)}_{\text {stiction }}+\underbrace{s_{d}^{2}\left(\omega_{m}\right)\left[s_{d}\left(\omega_{m}\right) T_{C}\left(\omega_{m}, T_{C}^{+}, T_{C}^{-}\right)+\beta \omega_{m}\right]}_{\text {Coulomb \& viscous }},
$$

with

$$
\begin{gathered}
\phi_{1}\left(\omega_{m}, u_{a}, T_{S}\right) \triangleq\left[1-s_{d}^{2}\left(\omega_{m}\right)\right] T_{F, S}\left(u_{a}, T_{S}\right) \\
\phi_{2}\left(\omega_{m}, T_{C}^{+}, T_{C}^{-}, \beta\right) \triangleq s_{d}^{2}\left(\omega_{m}\right)\left[s_{d}\left(\omega_{m}\right) T_{C}\left(\omega_{m}, T_{C}^{+}, T_{C}^{-}\right)+\beta \omega_{m}\right] .
\end{gathered}
$$

corresponding to the stiction and to the Coulomb-viscous friction components, respectively. The terms $T_{F, S}\left(u_{a}, T_{S}\right), s_{d}\left(\omega_{m}\right), T_{C}\left(\omega_{m}, T_{C}^{+}, T_{C}^{-}\right)$are defined in (2.9), (2.10) and (2.11). In the above expression we used the property $s_{d}^{2}\left(\omega_{m}\right)=1$ for $\left|\omega_{m}\right| \geq \omega_{t h}$. Figure 5 shows the simulated friction model and the Coulomb friction magnitude as a function of the motor angular velocity $\omega_{m}$.
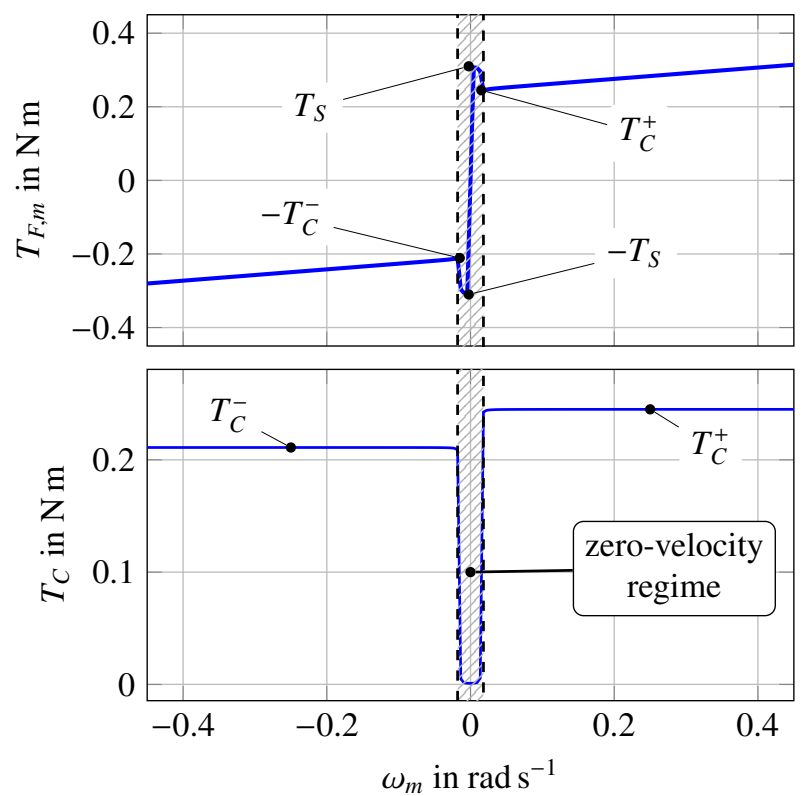

FIGURE 5 (Top) Motor friction as a function of the angular velocity. Stiction dominates for velocities very close to zero, while outside the "zero-velocity" area the only Coulomb and viscous friction contribute to the total frictional torque. (Bottom) Coulomb friction magnitude with different values for positive and negative velocities. Here, $T_{S}=0.31 \mathrm{~N} \mathrm{~m}, T_{C}^{+}=0.245 \mathrm{Nm}, T_{C}^{-}=$ $0.211 \mathrm{~N} \mathrm{~m}, \beta=0.154 \mathrm{~N} \mathrm{~s}, \omega_{t h}=0.015 \mathrm{~m} / \mathrm{sec}$.

Table 1 highlights the essential variables and parameters used in the smoothly parameterised model of friction.

\section{3 | Problem formulation}

The collective objective can be summarised in the following problem formulation: 
TABLE 1 System model nomenclature.

\begin{tabular}{clc}
\hline Symbol & Description & Units \\
\hline States, inputs and outputs & & $\mathrm{rad} \mathrm{s}^{-1}$ \\
\hline$\omega_{m}, \omega_{l}$ & Motor/load angular velocity & $\mathrm{rad}$ \\
$\theta_{m}, \theta_{l}$ & Motor/load angular position & $\mathrm{N} \mathrm{m}$ \\
$u$ & Torque generated by motor & - \\
\hline Constant parameters & & $\mathrm{kg} \mathrm{m}^{2}$ \\
\hline$N$ & Gearing ratio & $\mathrm{N} \mathrm{mad}^{-1}$ \\
$J_{m}, J_{l}$ & Motor/load inertia & $\mathrm{Nm} \mathrm{s} \mathrm{rad}^{-1}$ \\
$K_{S}$ & Shaft stiffness & $\mathrm{N} \mathrm{m}^{-1}$ \\
$D_{S}$ & Shaft damping coefficient & $\mathrm{N} \mathrm{m}^{-1}$ \\
$T_{C}^{+}, T_{C}^{-}$ & Coulomb friction magnitude & $\mathrm{rad} \mathrm{s}^{-1}$ \\
$T_{S}$ & Static friction & $\mathrm{N} \mathrm{m} \mathrm{s} \mathrm{rad}^{-1}$ \\
$\omega_{t h}$ & Zero-velocity threshold & - \\
$\beta$ & Viscous friction coefficient & \\
$p, \alpha$ & Signum/friction model slope & $\mathrm{N} \mathrm{m}$ \\
\hline Disturbances and developing torques & & $\mathrm{N} \mathrm{m}$ \\
\hline$T_{F, m}, T_{F, l}$ & Motor/load friction & \\
$T_{l}$ & Interconnecting torque & \\
\hline
\end{tabular}

Problem 1 (Friction parameters estimation). Given the single-axis drive-train system described in (2.1)-(2.5) and the friction model in (2.9), (2.10), (2.11) and (2.12), design an online dynamic estimator for the friction parameters $T_{S}, T_{C}^{+}, T_{C}^{-}, \beta$, such that each of the corresponding estimates $\hat{T}_{S}, \hat{T}_{C}^{+}, \hat{T}_{C}^{-}, \hat{\beta}$ convergence to a compact set containing the associated real parameter values $T_{S}^{*}, T_{C}^{+, *}, T_{C}^{-, *}, \beta^{*}$.

Remark 1. Notation. For a variable $\varrho$, the symbol $\hat{\varrho}$ denotes its estimate, $\tilde{\varrho} \triangleq \varrho-\hat{\rho}$ the associated estimation error and $\varrho^{*}$ a fixed numerical value that the variable assumes.

Remark 2. The models for the friction described earlier constitute an abstraction of the friction phenomenon itself. As a result, although the proposed parametrization captures the essential friction components, it is not exhaustive (e.g. the microscopic damping is not described). Therefore, the term "real friction parameters" refers to the values of the friction parameters associated to the proposed models that have been identified or calculated based on measurements on the experimental setup of this study.

Remark 3. As it will become clear in the following section, there is no restriction on which friction (on the motor or on the load) can be described and estimated with the proposed method. The motor friction has been chosen in this study as a test case.

Remark 4. The static and Coulomb friction coefficients $T_{S}, T_{C}^{+}, T_{C}^{-}$in Equations (2.9) and (2.11) are explicitly considered as arguments of the respective friction functions (stiction and Coulomb, respectively), as opposed to other parameters such as $p$ and $\alpha$. The reason for that is that the former three friction coefficients have to be estimated and can actually change over time due to wear. On the other hand, $\alpha$ and $p$ can be considered as tunable design parameters for the estimators.

\section{3 | FRICTION ESTIMATION}

The estimation scheme for the motor friction parameters comprises three distinct steps. The first step includes the task of estimating the total frictional torque acting on the drive motor. Once such an estimate is obtained, it can be partitioned into a stiction and a Coulomb-viscous component according to the zero/nonzero-velocity motion regimes. This is done during the second step. Finally, in the third step the two friction components are fed into two different adaptive estimators that provide online estimates of the friction parameters. The three steps are elaborated in the following. 


\section{1 | Estimation of the total friction}

From the dynamics of the drive motor in Equation (2.1), total friction torque $T_{F, m}$ is:

$$
T_{F, m}=u-\frac{1}{N} T_{l}-J_{m} \dot{\omega}_{m} .
$$

From Assumption 1 and since the input $u$ and measured outputs (angular positions and velocities) are available, the only term needed to calculate $T_{F, m}$ is an estimate of $\dot{\omega}_{m}$, i.e. of the angular acceleration of the drive motor.

Assumption 2. There exists a scalar locally Lipschitz function $f: \mathbb{R} \times \mathbb{R}^{4} \times \mathbb{R} \rightarrow \mathbb{R}$ such that the dynamical system

$$
J_{m} \dot{\hat{\omega}}_{m}=f\left(t, \boldsymbol{x}, \hat{\omega}_{m}\right), \hat{\omega}_{m}\left(t_{0}\right)=\omega_{m}\left(t_{0}\right)
$$

provides an estimate $\hat{\omega}_{m}$ of the motor angular velocity, with the associated estimation error $\tilde{\omega}_{m} \triangleq \omega_{m}-\hat{\omega}_{m}$ being bounded for all $t \geq t_{0}$.

A reasonable choice for $f$ in Equation (3.2) is:

$$
f\left(x, \hat{\omega}_{m}\right)=u-\frac{1}{N} T_{l}+K \tilde{\omega}_{m},
$$

where $T_{l}$ depends on the state vector $x$ and $K$ is a positive scalar gain. The resulting observer is a conventional linear one and the scheme (3.2),(3.3) results in a linear velocity differentiator with filtering provided by the observer. Note that the time argument in (3.3) has been omitted since this design comprises autonomous dynamics.

When Assumption 2 holds, then an estimate $\hat{T}_{F, m}$ of the total frictional torque on the drive motor is obtained from:

$$
\hat{\phi} \triangleq \hat{T}_{F, m}=\underbrace{u-\frac{1}{N} T_{l}}_{u_{a}}-J_{m} \dot{\hat{\omega}}_{m} .
$$

Since the term $\dot{\hat{\omega}}_{m}$ is always available, the bounded perturbation of the friction estimate is given by

$$
\tilde{T}_{F, m}=T_{F, m}-\hat{T}_{F, m}=-J_{m}\left(\dot{\omega}_{m}-\dot{\hat{\omega}}_{m}\right)=-J_{m} \dot{\tilde{\omega}}_{m} .
$$

From this it is easy to show that:

$$
\lim _{\tilde{\omega}_{m} \rightarrow 0} \hat{T}_{F, m}=T_{F, m} .
$$

Remark 5. If the focus should be on estimating the load friction parameters, then an observer for the load velocity could be employed instead, and the acceleration torque $u_{a}$ should be taken equal to the interconnecting torque $T_{l}$ (as can be easily deducted from the load dynamics equation).

\section{2 | Motion regime separation}

The second step of the method makes a partitioning of the total estimated friction into stiction and Coulomb-viscous components. Define two signals $\hat{\phi}_{1}$ and $\hat{\phi}_{1}$ as follows:

$$
\begin{aligned}
& \hat{\phi}_{1} \triangleq\left[1-s_{d}^{2}\left(\omega_{m}\right)\right] \hat{T}_{F, m} \\
& \hat{\phi}_{2} \triangleq s_{d}^{2}\left(\omega_{m}\right) \hat{T}_{F, m} .
\end{aligned}
$$

Proposition 1. Given an estimate $\hat{T}_{F, m}$ of total friction, the terms $\hat{\phi}_{1}$ and $\hat{\phi}_{2}$ respectively provide an estimation of the stiction and the Coulomb-viscous friction, with the associated estimation errors being bounded at all times.

Proof. When the motor is not moving $\left(\left|\omega_{m}\right|<\omega_{t h}\right)$, only stiction contributes to the total friction. Combining Equations (2.12), (3.5) and (3.6) and taking into account that $s_{d}^{2}\left(\omega_{m}\right)=0$ for $\left|\omega_{m}\right|<\omega_{t h}$, leads to

$$
\hat{\phi}_{1}=\phi_{1}+\underbrace{\left[1-s_{d}^{2}\left(\omega_{m}\right)\right] \tilde{T}_{F, m}}_{\tilde{\phi}_{1}}=\phi_{1}+\tilde{\phi}_{1}=T_{F, S}+\tilde{\phi}_{1},
$$

which is an estimation of the actual stiction perturbed by a bounded error coming from the total friction estimation. When the motor is moving, $s_{d}^{2}\left(\omega_{m}\right)=1$ and, hence, $\hat{\phi}_{1}=0$, which is equal to the actual stiction in the system. Following the same 
reasoning, one can easily show that

$$
\hat{\phi}_{2}=\phi_{2}+\underbrace{s_{d}^{2}\left(\omega_{m}\right) \tilde{T}_{F, m}}_{\tilde{\phi}_{2}}=\phi_{2}+\tilde{\phi}_{2}=s_{d}\left(\omega_{m}\right) T_{F, C}\left(\omega_{m}, T_{C}^{+}, T_{C}^{-}\right)+\beta \omega_{m}+\tilde{\phi}_{2} .
$$

When the motor is moving, $\left(\left|\omega_{m}\right|<\omega_{t h}\right)$, and $\hat{\phi}_{2}=0$ when is virtually immobile.

Remark 6. It should be noted that the estimation errors $\tilde{\phi}_{1}, \tilde{\phi}_{2}$ also account for modelling inaccuracies, especially related to parameter uncertainty and violation of Assumption 1.

Figure 6 shows the stiction and Figure 7 the Coulomb-viscous components, both of them obtained by multiplying the total friction in a simulation example with the terms $\left(1-s_{d}^{2}\left(\omega_{m}\right)\right)$ and $s_{d}^{2}\left(\omega_{m}\right)$, respectively. These two terms can be interpreted as decision functions with respect to whether the motor is moving or not.

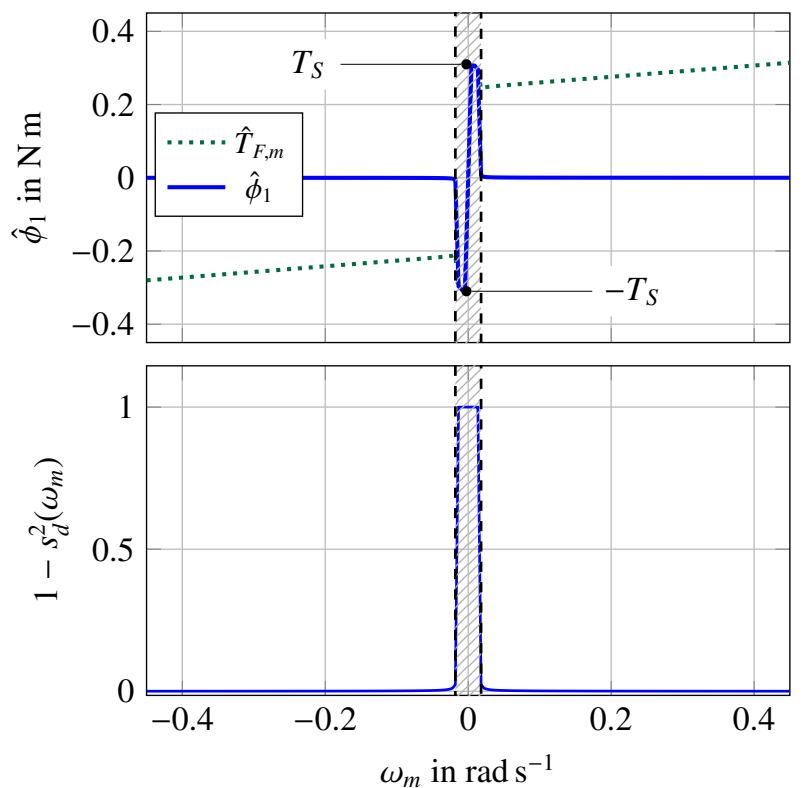

FIGURE 6 (Top) Stiction component extracted by an estimate of the total friction. The two signals overlap inside the $\pm \omega_{t h}$ region. (Bottom) Decision function for "zero-velocity" motion regime. Here, $T_{S}=0.31 \mathrm{~N} \mathrm{~m}, \omega_{t h}=0.015 \mathrm{~m} / \mathrm{sec}$.

\subsection{Adaptive estimators}

The last step of the proposed method includes the design of two separate adaptive estimators for the parameters of stiction and Coulomb-viscous friction components. Each of these estimators takes the corresponding estimated friction component as input and provides an estimate of the parameters associated with this component. The following considerations regarding the boundedness of the actuating torque $u_{a}$ and the set of the real friction parameters hold for the rest of the analysis:

1. The position and velocity differences between motor and load, respectively denoted by $\Delta \theta \triangleq \frac{\theta_{m}}{N}-\theta_{l}$ and $\Delta \omega \triangleq \frac{\omega_{m}}{N}-\omega_{l}$, are bounded signals, i.e. $(\Delta \theta, \Delta \omega) \in \mathcal{D} \subset \mathbb{R}^{2}$, where $\mathcal{D}$ is compact. This merely implies that the motor and the load stay connected at all times.

2. The control input $u$ belongs to the bounded set $V=\left[-u_{\max }, u_{\max }\right]$, where $u_{\max }$ is the maximum allowable torque that the motor can produce.

3. The static friction coefficient $T_{S}^{*}$ and its estimate $\hat{T}_{S}$ belong to the bounded set $S=\left(0, T_{S}^{\max }\right]$, where $T_{S}^{\max }$ denotes the maximum expected value for $T_{S}$. 


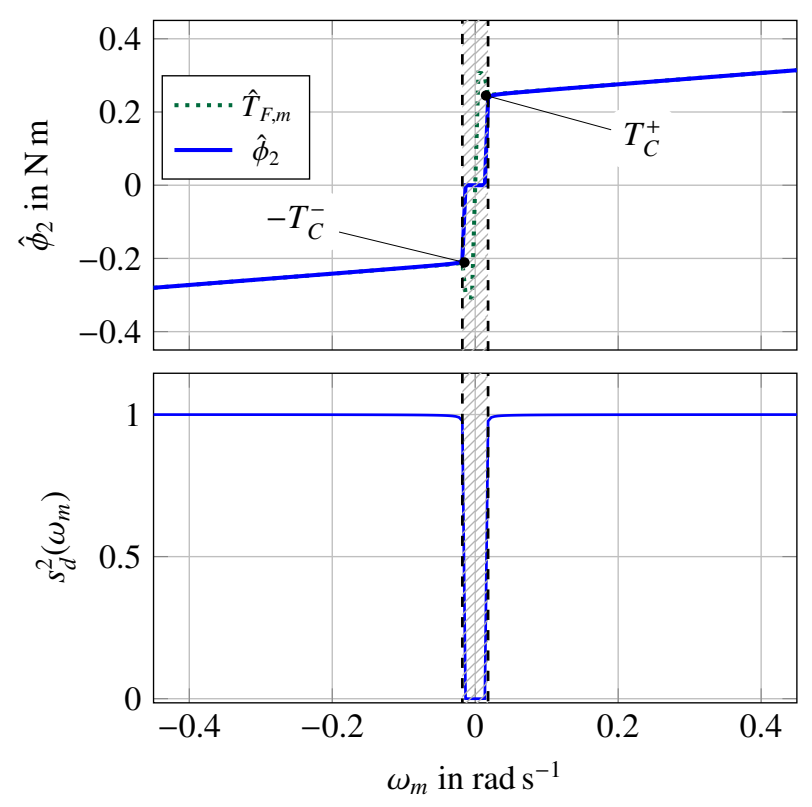

FIGURE 7 (Top) Coulomb and viscous friction component extracted by an estimate of the total friction. The two signals overlap outside the $\pm \omega_{t h}$ region. (Bottom) Decision function for "nonzero-velocity" motion regime. Here, $T_{C}^{+}=0.245 \mathrm{~N} \mathrm{~m}, T_{C}^{-}=$ $0.211 \mathrm{~N} \mathrm{~m}, \beta=0.154 \mathrm{~N} \mathrm{~s}, \omega_{t h}=0.015 \mathrm{~m} / \mathrm{sec}$.

4. The actual Coulomb and viscous friction parameters

$$
\vartheta^{*} \triangleq\left[\begin{array}{lll}
T_{C}^{+, *} & T_{C}^{-, *} & \beta^{*}
\end{array}\right]^{T}
$$

as well as their estimates $\hat{\vartheta}$ belong to the bounded set

$$
\Theta=\left(0, T_{C}^{+, \max }\right] \times \in\left(0, T_{C}^{-, \max }\right] \times \in\left(0, \beta^{\max }\right] .
$$

5. The physical parameters $T_{S}^{*}, \vartheta^{*}$ are constant or very slowly varying, i.e.

$$
\begin{aligned}
& \dot{T}_{S}^{*} \approx 0 \\
& \dot{\boldsymbol{\vartheta}}^{*} \approx \mathbf{0} .
\end{aligned}
$$

The superscript max denotes the maximum value a parameter can assume.

Remark 7. The first two assumptions ensure that the actuating torque $u_{a}$ remains bounded at all times. Moreover, the restriction of the parameter estimates to a bounded set can be achieved with the use of parameter projection. The Projection operator ${ }^{33}$ is defined in A.

The estimation of the static friction coefficient $T_{S}$ belongs to the class of problems of parameter identification in nonlinearly parameterised systems, for which several solutions have been reported in the literature $(34,35,36,37,38,39)$. The approach taken in this study is inspired by the design of a deadzone angle estimator presented in ${ }^{40}$, which is also based on the parameter identification method reported in $^{37}$. The design of the estimator is detailed in the following proposition.

Proposition 2 (Static friction coefficient estimator). Consider the stiction model defined in Equation (2.13) and an estimate $\hat{\phi}_{1}$ of the motor stiction $\phi_{1}$ with bounded associated estimation error $\tilde{\phi}_{1}=\phi_{1}-\hat{\phi}_{1}$. Consider also two real non-negative functions $\mu\left(u_{a}, \hat{T}_{S}\right): V \times \mathcal{D} \times S \rightarrow \mathbb{R}_{\geq 0}$ and $\sigma\left(u_{a}\right): V \times \mathcal{D} \rightarrow \mathbb{R}_{\geq 0}$ with the following properties:

$$
\begin{aligned}
\mu\left(u_{a}, T_{S}^{A}\right) \frac{\partial T_{F, S}}{\partial T_{S}}\left(u_{a}, T_{S}^{B}\right) & \geq \sigma\left(u_{a}\right) \\
\left|T_{F, S}\left(u_{a}, T_{S}^{B}\right)-T_{F, S}\left(u_{a}, T_{S}^{A}\right)\right| & \leq L \sqrt{\sigma\left(u_{a}\right)}\left|T_{S}^{B}-T_{S}^{A}\right|
\end{aligned}
$$


for any fixed $u_{a} \in \mathcal{V} \times \mathcal{D}$ and all pairs $\left(T_{S}^{A}, T_{S}^{B}\right) \in S \times S$, where $L$ is a positive real constant. Let $\tilde{T}_{S} \triangleq T_{S}^{*}-\hat{T}_{S}$ be the static friction coefficient estimation error. Provided that the following Persistence of Excitation (PE) condition

$$
\int_{t}^{t+T} \sigma\left(u_{a}(\tau)\right) d \tau \geq \varepsilon, \forall t \in \mathbb{R}_{\geq 0}
$$

holds and for some $\varepsilon, T>0$, the adaptive estimator

$$
\dot{\hat{T}}_{S}=\operatorname{Proj}\left[\hat{T}_{S}, \gamma \mu\left(u_{a}, \hat{T}_{S}\right)\left(\hat{\phi}_{1}-\phi_{1}\left(u_{a}, \hat{T}_{S}\right)\right)\right], \gamma>0
$$

where $\gamma$ is the adaptation gain, guarantees that:

1. The origin $\tilde{T}_{S}^{*}=0$ is a Uniformly Locally Exponentially Stable (ULES) equilibrium point of the $\tilde{T}_{S}$-dynamics if $\hat{\phi}_{1}$ is an exact estimate of the stiction, i.e. if $\hat{\phi}_{1}=\phi_{1}\left(u_{a}, T_{S}\right)$.

2. The origin $\tilde{T}_{S}^{*}=0$ is a Uniformly Globally Asymptotically Stable (UGAS) equilibrium point of the $\tilde{T}_{S}$-dynamics if $\hat{\phi}_{1}$ is an UGAS estimate of the actual stiction.

3. The estimation error $\tilde{T}_{S}$ is Uniformly Globally Bounded (UGB) if $\left|\tilde{\phi}_{1}\right|<\Phi_{1}$, where $\Phi_{1}$ is a positive finite number.

\section{Proof. See Appendix B.}

Selecting the functions $\mu, \sigma$ as

$$
\begin{gathered}
\mu\left(u_{a}, \hat{T}_{S}\right) \triangleq \frac{u_{a} \pi}{\chi\left(u_{a}, \hat{T}_{S}\right)+\epsilon} \\
\sigma\left(u_{a}\right) \triangleq u_{a} \chi\left(u_{a}, E\right)
\end{gathered}
$$

where

$$
\chi\left(u_{a}, y\right) \triangleq \frac{\alpha}{1+\alpha^{2}\left(u_{a}+y\right)^{2}}+\frac{\alpha}{1+\alpha^{2}\left(u_{a}-y\right)^{2}},
$$

$\epsilon>0$ is a small positive number and $E>T_{S}^{\max }$, ensures that properties (3.10) and (3.11) are satisfied everywhere in $V \times D \times S \times S$ as illustrated in Figure 8 . This can be easily shown analytically by showing that the function $\frac{\partial T_{F, S}}{\partial \hat{T}_{S}}$ is strictly decreasing in the interval $\left(u_{a}, T_{S}^{\max }\right]$ for any given $u_{a}$.

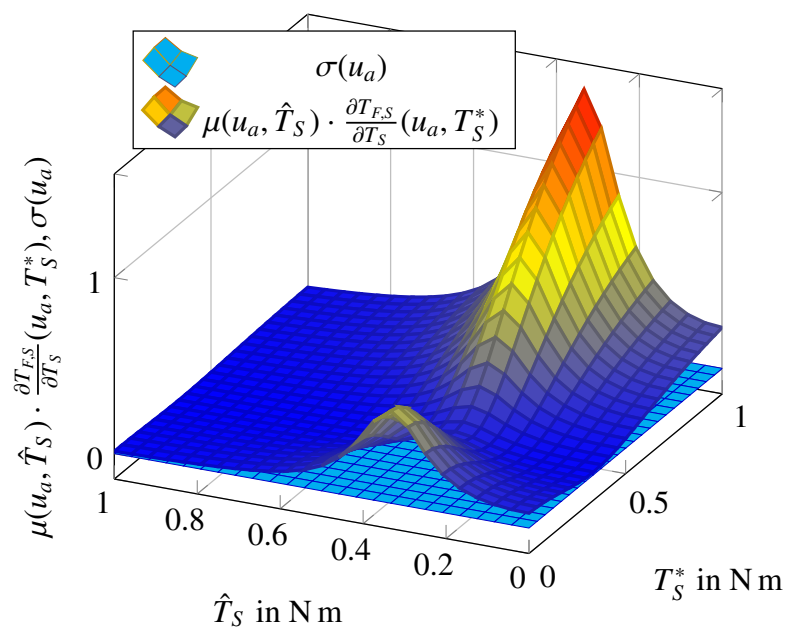

FIGURE 8 Graphical representation of Property (3.10) for a fixed value of $u_{a}$. As it can be seen, the surface $z=\mu\left(u_{a}, \hat{T}_{S}\right)$. $\frac{\partial T_{F, S}}{\partial T_{S}}\left(u_{a}, T_{S}^{*}\right)$ corresponding to the right part of the inequality (3.10) is always above the plane $z=\sigma\left(u_{a}\right)$, for all pairs $\left(\hat{T}_{S}, T_{S}^{*}\right) \in$ $(0,1] \times(0,1]$. 
Remark 8. The specific selection of $\mu, \sigma$ ensure boundedness of the functions in the sets $V \times \mathcal{D} \times S$ and $V \times D$, respectively.

The partial derivative of $T_{F, S}$ with respect to $\hat{T}_{S}$ is given by

$$
\frac{\partial T_{F, S}}{\partial \hat{T}_{S}}=\frac{u_{a}}{\pi} \chi\left(u_{a}, \hat{T}_{S}\right)
$$

and it is bounded everywhere in $V \times D \times S$. Then there exists positive constant $L_{0}$, such that

$$
\left|T_{F, S}\left(u_{a}, T_{S}^{B}\right)-T_{F, S}\left(u_{a}, T_{S}^{A}\right)\right| \leq L_{0}\left|T_{S}^{B}-T_{S}^{A}\right|=\frac{L_{0}}{\sqrt{\sigma\left(u_{a}\right)}} \sqrt{\sigma\left(u_{a}\right)}\left|T_{S}^{B}-T_{S}^{A}\right|<L \sqrt{\sigma\left(u_{a}\right)}\left|T_{S}^{B}-T_{S}^{A}\right|
$$

for $u_{a} \neq 0$ with $L \triangleq L_{0} / \sqrt{\varsigma}$. If $u_{a}=0$, then $T_{F, S}\left(u_{a}, T_{S}^{B}\right)=T_{F, S}\left(u_{a}, T_{S}^{A}\right)=0$, therefore the inequality holds by default and, hence, Property (3.11) is satisfied everywhere in $V \times \mathcal{D} \times S \times S$.

Proposition 3 (Coulomb and viscous friction coefficients estimator). Consider the Coulomb and viscous friction model defined in Equation (2.14) and an estimate $\hat{\phi}_{2}$ of the motor Coulomb and viscous friction $\phi_{2}$ with bounded associated estimation error $\tilde{\phi}_{2}=\phi_{2}-\hat{\phi}_{2}$. The adaptive estimator

$$
\dot{\hat{\boldsymbol{\vartheta}}}=\Gamma \boldsymbol{\psi}\left(\omega_{m}\right)\left(\hat{\phi}_{2}-\phi_{2}\left(\omega_{m}, \hat{\boldsymbol{\vartheta}}\right)\right),
$$

where $\Gamma$ is a positive definite matrix, $\phi_{2}\left(\omega_{m}, \hat{\boldsymbol{\vartheta}}\right)$ is the reconstructed Coulomb and viscous friction torque and $\boldsymbol{\psi}\left(\omega_{m}\right), \hat{\boldsymbol{\vartheta}}$ are defined as

$$
\begin{aligned}
\boldsymbol{\psi}\left(\omega_{m}\right) & \triangleq\left[\begin{array}{c}
s_{d}\left(\omega_{m}\right)\left(\frac{1}{2}+\frac{1}{\pi} \tan ^{-1}\left(\alpha\left(\omega_{m}-\omega_{t h}\right)\right)\right) \\
s_{d}\left(\omega_{m}\right)\left(\frac{1}{2}-\frac{1}{\pi} \tan ^{-1}\left(\alpha\left(\omega_{m}+\omega_{t h}\right)\right)\right) \\
\omega_{m}
\end{array}\right] \\
\hat{\boldsymbol{\vartheta}} & \triangleq\left[\begin{array}{lll}
\hat{T}_{C}^{+} & \hat{T}_{C}^{-} & \hat{\beta}
\end{array}\right]^{T}
\end{aligned}
$$

guarantees that under the following PE condition

$$
\int_{t}^{t+T} \boldsymbol{\psi}\left(\omega_{m}(\tau)\right) \boldsymbol{\Psi}^{T}\left(\omega_{m}(\tau)\right) d \tau \geq n \boldsymbol{I}, \forall t \in \mathbb{R}_{\geq 0} n, T>0
$$

with excitation level $n$, the parameter estimation error $\tilde{\boldsymbol{\vartheta}} \triangleq \boldsymbol{\vartheta}^{*}-\hat{\boldsymbol{\vartheta}}$ remains bounded for all future times. If, additionally, $\tilde{\phi}_{2}=0$, then

$$
\lim _{t \rightarrow \infty} \tilde{\vartheta}(t)=\mathbf{0} .
$$

Proof. Similarly to the proof of Proposition 2, the analysis is only done for the case $\left|\omega_{m}\right| \geq \omega_{t h}$, the region where Coulomb and viscous are identifiable $\left(\hat{\phi}_{2}=0\right.$ for $\left.\left|\omega_{m}\right|<\omega_{t h}\right)$. Consider the smooth positive definite Lyapunov function candidate

$$
V_{2}(\tilde{\boldsymbol{\vartheta}})=\frac{1}{2} \tilde{\vartheta}^{T} \Gamma^{-1} \tilde{\boldsymbol{\vartheta}} .
$$

Its time derivative along the dynamics of the estimation error $\tilde{\vartheta}$ reads

$$
\begin{aligned}
\dot{V}_{2}(\tilde{\boldsymbol{\vartheta}}) & =\tilde{\boldsymbol{\vartheta}}^{T} \boldsymbol{\Gamma}^{-1} \dot{\tilde{\boldsymbol{\vartheta}}}=-\tilde{\boldsymbol{\vartheta}}^{T} \boldsymbol{\Gamma}^{-1} \dot{\hat{\boldsymbol{\vartheta}}}=-\tilde{\boldsymbol{\vartheta}}^{T} \boldsymbol{\psi}\left(\omega_{m}\right)\left(\hat{\phi}_{2}-\phi_{2}\left(\omega_{m}, \hat{\boldsymbol{\vartheta}}\right)\right)=-\tilde{\boldsymbol{\vartheta}}^{T} \boldsymbol{\psi}\left(\omega_{m}\right)\left(\phi_{2}\left(\omega_{m}, \boldsymbol{\vartheta}\right)-\phi_{2}\left(\omega_{m}, \hat{\boldsymbol{\vartheta}}\right)-\tilde{\phi}_{2}\right)= \\
& =-\tilde{\boldsymbol{\vartheta}}^{T} \boldsymbol{\psi}\left(\omega_{m}\right) \boldsymbol{\psi}^{T}\left(\omega_{m}\right) \tilde{\boldsymbol{\vartheta}}+\tilde{\boldsymbol{\vartheta}}^{T} \boldsymbol{\psi}\left(\omega_{m}\right) \tilde{\phi}_{2} \leq-\|\tilde{\boldsymbol{\vartheta}}\|^{2}\left\|\boldsymbol{\psi}\left(\omega_{m}\right) \boldsymbol{\psi}^{T}\left(\omega_{m}\right)\right\|+\|\tilde{\boldsymbol{\vartheta}}\| \cdot\left\|\boldsymbol{\psi}\left(\omega_{m}\right)\right\| \cdot\left|\tilde{\phi}_{2}\right|= \\
& =-(1-q)\|\tilde{\boldsymbol{\vartheta}}\|^{2}\left\|\boldsymbol{\psi}\left(\omega_{m}\right) \boldsymbol{\psi}^{T}\left(\omega_{m}\right)\right\|+\|\tilde{\boldsymbol{\vartheta}}\|\left(\left\|\boldsymbol{\psi}\left(\omega_{m}\right)\right\| \cdot\left|\tilde{\phi}_{2}\right|-q\|\tilde{\boldsymbol{\vartheta}}\| \cdot\left\|\boldsymbol{\psi}\left(\omega_{m}\right) \boldsymbol{\psi}^{T}\left(\omega_{m}\right)\right\|\right) \leq \\
& \leq-(1-q)\|\tilde{\boldsymbol{\vartheta}}\|^{2}\left\|\boldsymbol{\psi}\left(\omega_{m}\right) \boldsymbol{\psi}^{T}\left(\omega_{m}\right)\right\| .
\end{aligned}
$$

for

$$
\|\tilde{\vartheta}\|>\frac{\left\|\boldsymbol{\psi}\left(\omega_{m}\right)\right\|}{q\left\|\boldsymbol{\psi}\left(\omega_{m}\right) \boldsymbol{\Psi}^{T}\left(\omega_{m}\right)\right\|}\left|\tilde{\phi}_{2}\right|
$$

where $0<q<1$. From (3.22), (3.23) it can be seen that $V_{2}$ is non-increasing outside a ball of radius proportional to the norm of the estimation error $\tilde{\phi}_{2}$. This implies that $\tilde{\boldsymbol{\vartheta}}$ remains bounded for all future times, provided that $\tilde{\phi}_{2}$ is bounded. The condition $\left\|\boldsymbol{\psi}\left(\omega_{m}\right) \boldsymbol{\Psi}^{T}\left(\omega_{m}\right)\right\| \neq 0$ is ensured from the fact that in this motion regime $\omega_{m} \neq 0$ and hence, $\boldsymbol{\psi}\left(\omega_{m}\right) \neq \mathbf{0}$. 
If now $\tilde{\phi}_{2}$ asymptotically converges to 0 , then by using the same arguments as in the proof of Proposition 2 , it can be shown that $\tilde{\boldsymbol{\vartheta}}$ converges to 0 as time goes to infinity. It should be noted that if $\tilde{\phi}_{2}=0$, boundedness of the parameter estimation error is always guaranteed even without the PE condition (follows from application of Barbalat's lemma for the unperturbed estimation error system).

Remark 9. The structure of the friction estimation scheme is modular in the sense that the three steps can be designed and tuned separately. This means that for the estimation of the motor angular acceleration, any type of velocity observer or differentiator can be used (see ${ }^{40}$ where a Super-Twisting Sliding-Mode Observer (STSMO) was used instead), so long the associated acceleration estimation error is bounded. This design flexibility is the main difference from the method proposed in ${ }^{37}$, where the problem of obtaining the perturbation that drives the adaptive estimator is not decoupled from the parameter estimation itself.

The entire friction estimation scheme is summarised in the following steps and illustrated in Figure 9.
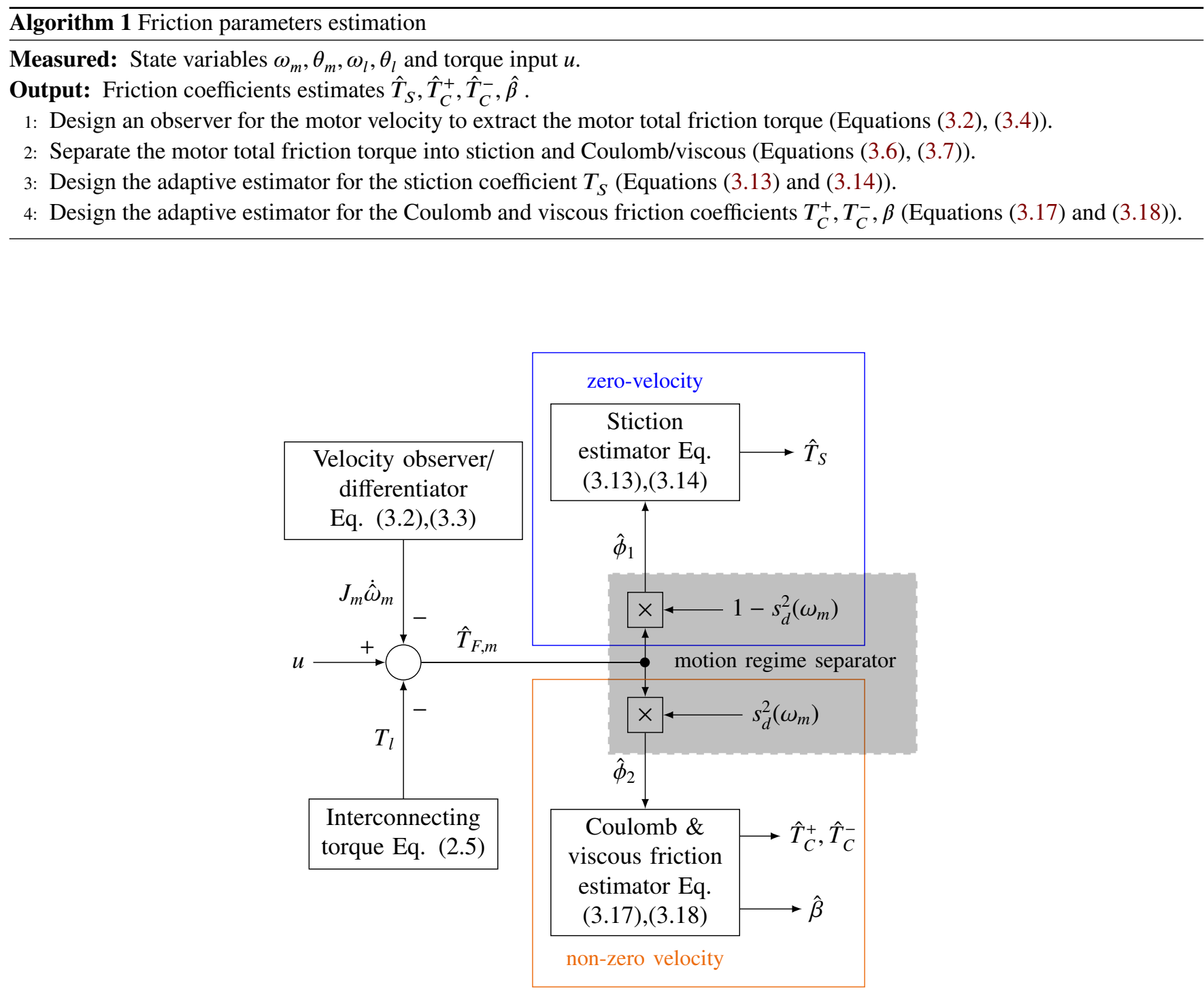

FIGURE 9 Block diagram of the estimation scheme. 


\section{4 | EXPERIMENTAL RESULTS}

\section{1 | Experimental setup}

The friction estimation algorithm was tested on a drive-train consisting of two Siemens 1FT7042-5AF70 permanent magnet synchronous motors (PMSMs) connected through a steel shaft. One motor is the drive, the other simulates static and dynamic loads. The experimental setup was equipped with a Siemens SINAMICS S120 drive converters with 11-bit IC22DQ incremental angle encoders. With the encoder quantisation levels approximately at $1.5 \mu \mathrm{rad}$, the noise in the position measurements could be neglected. However, velocity measurements, obtained via numerical differentiation already embedded in the S120 firmware, were inflicted with noise that was approximated as white Gaussian with $\sigma_{\omega} \approx 9 \cdot 10^{-3} \mathrm{rad} \mathrm{s}^{-1}$ as shown in Figure 10. A cylindrical Vari-tork 279.25 .22 clutch $^{41}$ was mounted on a steel base and around the interconnecting shaft to adjust the motor friction through a rotating ring. The entire physical test rig is depicted in Figure 11.
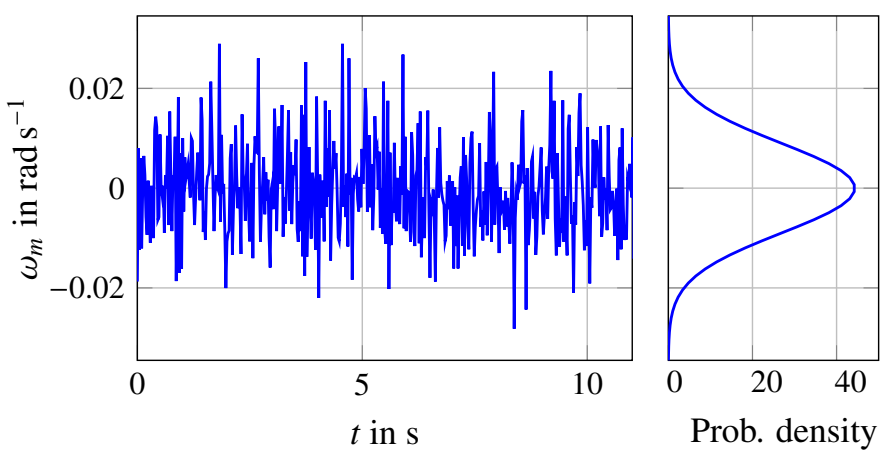

FIGURE 10 (Left) Drive motor zero velocity measurement. (Right) Probability distribution of velocity measurement noise.

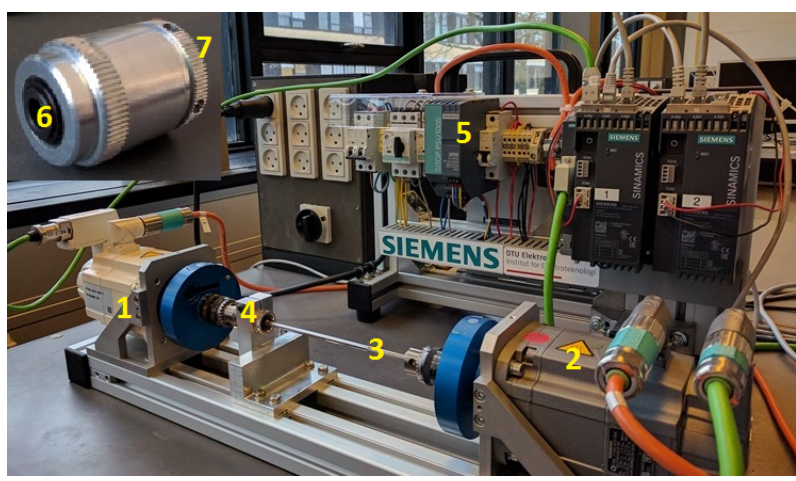

FIGURE 11 Experimental setup: (1) 1FT7 drive PMSM, (2) 1FT7 load PMSM, (3) shaft, (4) friction component base, (5) Siemens SINAMICS S120 converter, (6) shaft housing, (7) friction adjustment ring.

\section{2 | Test scenarios}

The drive motor was position-controlled to track a slow sinusoidal reference signal $\theta_{r}(t)=\sin (0.2 \pi t)$. The selection of this motion pattern was based on the fact that all tool tip trajectories in machine tool applications are composed of closed curves and large segments of the tool path consist of periodic trajectories. Therefore motion profiles that include axis reversals are guaranteed during machine tool operation. The friction estimators were tested in four different friction scenarios of $500 \mathrm{~s}$ each. The different test cases corresponding to four positions of the friction adjustment ring (0,90,180 and 360 degrees) are listed in 
Table 2. In each of this cases, only Coulomb friction and stiction were varied. The corresponding values for these parameters were measured using a torque meter. The viscous friction coefficient was identified during constant velocity experiments. An additional test at higher speeds with nominal friciton values (with the friction adjustment ring not mounted) was run for testing the estimation scheme with respect to rich excitation of viscous friction. A linear Luenbeger velocity observer was used to estimate the total friction torque acting on the drive motor. The real system parameters, which were identified offline, as well as the estimators' and the observer gains are shown in Table 3.

TABLE 2 Test scenarios.

\begin{tabular}{lcccc}
\hline Test & $T_{S}^{*}(\mathrm{~N} \mathrm{~m})$ & $T_{C}^{+, *}(\mathrm{~N} \mathrm{~m})$ & $T_{C}^{-, *}(\mathrm{~N} \mathrm{~m})$ & $\beta^{*}\left(\mathrm{~N} \mathrm{~m} \mathrm{~s} \mathrm{rad}^{-1}\right)$ \\
\hline$T_{1}$ & 0.2668 & 0.1424 & 0.1380 & 0.0016 \\
$T_{2}$ & 0.2712 & 0.1492 & 0.1289 & 0.0016 \\
$T_{3}$ & 0.5747 & 0.4817 & 0.4605 & 0.0016 \\
$T_{4}$ & 0.7709 & 0.6787 & 0.6138 & 0.0016 \\
\hline \multicolumn{4}{r}{ Higher-velocity test with nominal friction and $\theta_{r}(t)=\sin (6 \pi t)$} & 0.0016 \\
\hline$T_{5}$ & 0.0313 & 0.021 & 0.017 & \\
\hline
\end{tabular}

TABLE 3 System and estimators' parameter values.

\begin{tabular}{clc}
\hline Symbol & Description & Value \\
\hline Physical system parameters & & \\
\hline$J_{m}$ & Inertia & $0.000831 \mathrm{~kg} \mathrm{~m}^{2}$ \\
$K_{S}$ & Shaft stiffness & $31.75 \mathrm{~N} \mathrm{mad}^{-1}$ \\
$D_{S}$ & Shaft damping coefficient & $0.054 \mathrm{~N} \mathrm{~m} \mathrm{sad}^{-1}$ \\
$N$ & Gearing ratio & 1 \\
\hline Velocity observer parameters & & 275 \\
\hline$K$ & Observer gain & \\
\hline Estimators parameters & & $5 \cdot 10^{5}$ \\
\hline$\alpha$ & Smoothness factor (friction) & $10^{4}$ \\
$p$ & Smoothenss factor (signum) & $0.2 \mathrm{rad} \mathrm{s}^{-1}$ \\
$\omega_{t h}$ & Zero-velocity threshold & 0.5 \\
$\gamma$ & $\hat{T}_{S}$ adaptation gain & {$[0.15,0.15,0.005]$} \\
diag $(\boldsymbol{\Gamma})$ & $\hat{\boldsymbol{\vartheta}}$ adaptation gain & $0.008 \mathrm{Nm}$ \\
$\hat{T}_{S, 0}$ & Initial conditions & $1.25 \mathrm{Nm}$ \\
$T_{S}^{\max }$ & Maximum Stiction coefficient & {$[0.008,0.008,0.01]^{T}$} \\
$\hat{\vartheta}_{\mathbf{0}}$ & Initial conditions & {$[1.25,1.25,0.1]^{T}$} \\
$\boldsymbol{\vartheta}^{\text {max }}$ & Maximum Coulomb/viscous coefficients & \\
\hline
\end{tabular}




\section{3 | Results}

The metrics used for evaluation of the estimator performance are the Maximum Absolute Estimation Error (MAEE) and the Relative Maximum Absolute Estimation Error (RMAEE) defined as ${ }^{40}$ :

$$
\begin{aligned}
M A E E & =\max _{t_{0} \leq t \leq t_{0}+T}|\tilde{\eta}(t)| \\
R M A E E & =100 \frac{M A E E}{\eta} \%,
\end{aligned}
$$

where $\eta$ denotes any of the four friction parameters and the interval $\left[t_{0}, t_{0}+T\right]$ corresponds to the last 20 seconds of logged data. Each experiment had a duration of 500s, equivalent to a 10-cycles time window after all transients died out. The MAEE and RMAEE of all the parameters for the five tests are shown in Table 4, where RMAEE is denoted by $e_{i}$ with $i$ being the subscript of the associated parameter. The Table shows that the static friction coefficient error is smaller than $1 \%$ of its actual value in all cases, and the Coulomb friction estimation errors are below $17 \%$ of actual values. This can be also seen in Figures 12,13 and 14, which show the estimated total friction torque, stiction and the Coulomb-viscous friction, along with the values reconstructed via the model with estimated parameters and subjected to actual input. The reconstructed friction components match very closely with actual torque signals even though the viscous coefficient estimation error is large. Table 5 shows the MAEE of the four parameters as a percentage of the rated torque of the drive motor $T_{\text {rated }}=2.7 \mathrm{~N} \mathrm{~m}^{42}$, i.e. the quantities $100 \frac{M A E E}{T_{\text {rated }}} \%$. In the worst cases these percentages do not exceed $1.21 \%$. The estimate of the viscous friction coefficient at low speeds (tests $T_{1}-T_{4}$ ), is far from the measured value. This is expected since the excitation levels for this parameter are very small during the operation cycle. Overall this means that the influence of viscous friction is tiny compared to total friction at such low speeds ( $<1 \mathrm{rad} / \mathrm{s}$ ). Segments of manufacturing cycles with faster reference signal variation will favour the estimation of the viscous friction coefficient at the expense of reduced excitation richness for the stiction. This is confirmed by the last test $\left(T_{5}\right)$ at higher velocity. As it can be seen in Tables 4, 5 and Figure 15, the viscous friciton coefficient $\beta_{m}$ is estimated at higher velocities with $0.84 \%$ relative estimation error with respect to the actual value, which corresponds to approximately $0.0005 \%$ of the motor's rated torque.

The time responses of the estimation errors for the four parameters and the four test cases are shown in Figures 16-19 and 20. It can be seen that the small peaks patterns of the motor acceleration error, i.e. of the friction torque estimation error, appear in the parameter estimates. This is expected after considering the adaptation laws (3.13) and (3.17). Finally the velocity-friction plots for the four tests are shown in Figure 21 which illustrates the estimated friction and the reconstructed one based on the estimated parameters. The fit was calculated as

$$
f i t=100 \cdot \sqrt{\frac{\sum_{1}^{N_{s}}\left(\hat{T}_{F, m}^{i}-T_{F, m}^{i}\left(\hat{T}_{S}, \hat{\vartheta}\right)\right)^{2}}{\sum_{1}^{N_{s}}\left(\hat{T}_{F, m}^{i}-\frac{1}{N_{s}} \sum_{1}^{N_{s}} \hat{T}_{F, m}^{i}\right)^{2}}},
$$

where $N_{s}$ is the number of samples and the superscript $i$ denotes the $i^{\text {th }}$ sample of the associated signal. A similar plot is shown in Figure 22, where the friction torque is reconstructed based on both the real values of the coefficients (blue line) and the ones estimated by the proposed scheme (red line). Figure 22 essentially does not take into account the discrepancy between the real frictional phenomena and the description provided by the used model. As seen, the fit of the reconstructed friction torques to the ones estimated by observer is better for larger friction coefficients. This can be explained by the fact that in such cases the friction torque is larger than any other torque perturbation due to parameter model uncertainty and the electrical dynamics of the motor, and can be separated from them more effectively. This means the observer-based torque estimation error $\tilde{\phi}$ is much smaller than the friction torque itself.

\section{5 | DISCUSSION}

The main structural idea in the proposed estimation scheme pertains to decomposing the estimated total friction torque into two constituents that do not overlap with each other. Indeed, estimation of the four friction parameters we consider might not necessarily require inclusion of the nonlinear friction dynamics in the description of the phenomenon. This is due to the fact that at speeds faster than a threshold the dominant components of friction, i.e. Coulomb and viscous friction, are sufficiently 
TABLE 4 MAEE in N $m$ and RMAEE for all five tests.

\begin{tabular}{ccccccccc}
\hline Test & $\tilde{T}_{S}(\mathrm{Nm})$ & $e_{S}(\%)$ & $\tilde{T}_{C}^{+}(\mathrm{Nm})$ & $e_{C}^{+}(\%)$ & $\tilde{T}_{C}^{-}(\mathrm{N} \mathrm{m})$ & $e_{C}^{-}(\%)$ & $\tilde{\beta}\left(\mathrm{N} \mathrm{m} \mathrm{s} \mathrm{rad}^{-1}\right)$ & $e_{\beta}(\%)$ \\
\hline$T_{1}$ & 0.0008 & 0.3 & 0.0223 & 15.6 & 0.0070 & 5.07 & 0.0016 & 100 \\
$T_{2}$ & 0.0023 & 0.85 & 0.0221 & 14.8 & 0.0210 & 16.29 & 0.0016 & 100 \\
$T_{3}$ & 0.0051 & 0.89 & 0.0055 & 1.14 & 0.0167 & 3.63 & 0.0128 & 800 \\
$T_{4}$ & 0.0015 & 0.19 & 0.0062 & 0.91 & 0.0326 & 5.31 & 0.0205 & 1281 \\
\hline \multicolumn{7}{c}{ Higher-velocity test with nominal friction and $\theta_{r}(t)=\sin (6 \pi t)$} \\
\hline$T_{5}$ & 0.0313 & 100 & 0.0015 & 7.14 & 0.0073 & 42.94 & 0.0000135 & 0.84 \\
\hline
\end{tabular}

TABLE 5 MAEE as a percentage of the rated torque $(2.7 \mathrm{Nm})$ of the drive motor for all five tests.

\begin{tabular}{|c|c|c|c|c|}
\hline Test & $\tilde{T}_{S}(\%)$ & $\tilde{T}_{C}^{+}(\%)$ & $\tilde{T}_{C}^{-}(\%)$ & $\tilde{\beta}(\%)$ \\
\hline$\overline{T_{1}}$ & 0.0296 & 0.8259 & 0.2593 & 0.0593 \\
\hline$T_{2}$ & 0.0852 & 0.8185 & 0.7778 & 0.0593 \\
\hline$T_{3}$ & 0.1889 & 0.2307 & 0.6185 & 0.4741 \\
\hline$T_{4}$ & 0.0556 & 0.2296 & 1.2074 & 0.7593 \\
\hline \multicolumn{5}{|c|}{ Higher-velocity test with nominal friction and $\theta_{r}(t)=\sin (6 \pi t)$} \\
\hline$T T_{5}$ & 1.1593 & 0.0556 & 0.2704 & 0.0005 \\
\hline
\end{tabular}

described by simple static models. On the other hand, at low speeds, where the nonlinearities dominate, it is only of interest to obtain a "limit" description of the developing friction, which corresponds to the static friction coefficient. This segregation of the two types of friction allows for a separate design of the estimators, which are completely independent of each other. This, together with the fact that the total friction is obtained by an observer that guarantees a bounded estimation error, constitutes a modular architecture, which facilitates independent tuning and optimisation of each part of the estimation scheme.

Efficient separation of the two motion regimes (zero and nonzero speed) requires to select the threshold velocity $\omega_{t h}$. A reasonable initial choice is the $3 \sigma_{\omega}$ value of noise on the velocity signal when the motor is immobile.

Apart from the velocity observer accuracy, robustness of the estimation scheme also depends on the model mismatches and parameters uncertainty, as well as on the selection of the smoothness factors $\alpha$ and $p$. Typically, these parameters are chosen to be large positive numbers ( $>1000$ ), such that the approximation of the discontinuities is as good as possible. Higher values for $\alpha$ and $p$ lead to better approximation but also to reduced sensitivity of the stiction to the static friction coefficient $T_{S}$, which implies slower convergence. This can be seen by examining the partial derivative of $T_{F, S}$ with respect to $\hat{T}_{S}$ in Equation (3.16), which reveals an inverse proportionality of the sensitivity function to the smoothing factor $\alpha$. Figure 23 illustrates the approximation error in the stiction modelling as a function of $\alpha$, captured by the difference

$$
\begin{aligned}
\delta_{S} & \triangleq \frac{100}{u_{a}^{\max }} \cdot \max _{u_{a}}\left|\delta_{1}-\delta_{2}\right|, \text { with } \\
\delta_{1} & =\frac{1}{2}\left[1-\operatorname{sgn}\left(\left|u_{a}\right|-T_{S}\right)\right] \\
\delta_{2} & =\frac{1}{\pi}\left[\tan ^{-1}\left(\alpha\left(u_{a}+T_{S}\right)\right)-\tan ^{-1}\left(\alpha\left(u_{a}-T_{S}\right)\right)\right]
\end{aligned}
$$

which is a percentage of the maximum actuating torque $u_{a}^{\max }$. The error is reduced for increasing $\alpha$ at almost exponential rate.

Regarding the tuning of the estimators, the adaptation gains $\gamma, \boldsymbol{\Gamma}$ reflect the trade-off between fast parameter convergence and robustness to sudden unmodelled disturbances and noise in the system. A good way to select these gains is to start from small values, close to the reciprocal of the regressor function maximum absolute value (or the $\mu$ function in the case of stiction), and then increase them until the desired convergence rate is achieved. The gains can also be scheduled according to the operation conditions, i.e. to the levels of excitation (e.g. use smaller value for $\gamma$ when the speeds are much lower than in the initial tuning experiment). One last possible refinement of the algorithm pertains to the normalization of the parameters by scaling them by 

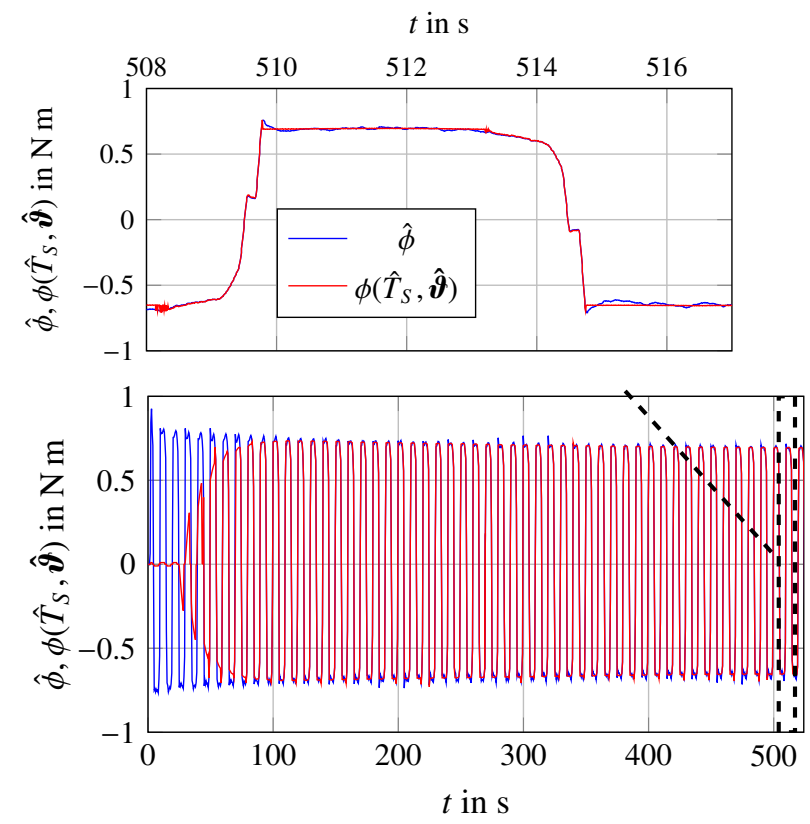

FIGURE 12 Total friction torque $\hat{\phi}$ and its reconstructed counterpart $\phi\left(\hat{T}_{S}, \hat{\vartheta}\right)$ based on the friction parameters estimates.
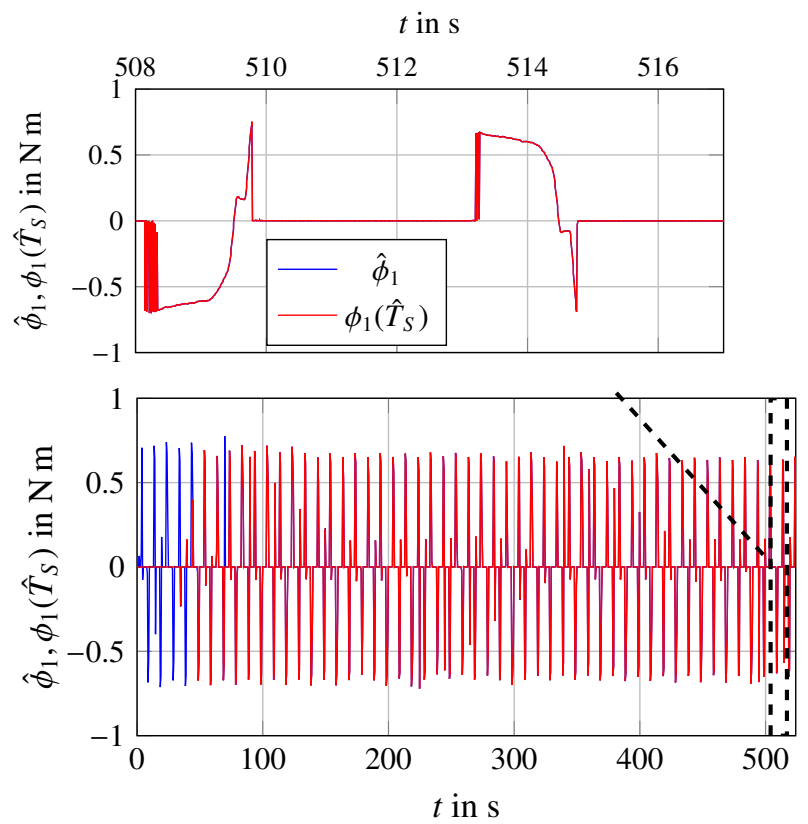

FIGURE 13 Static friction (stiction) $\hat{\phi}_{1}$ and its reconstructed counterpart $\phi_{1}\left(\hat{T}_{S}\right)$ based on the static friction coefficient estimate.

their maximum value. This will make the implementation of the projection easier, since all parameter estimates will be restricted within the unit ball instead of a fourth-dimensional ellipsoid.

\section{6 | CONCLUSIONS}

This paper developed a modular online friction estimation scheme for machine tools based on motion-regime separation. The suggested method utilised estimation of total frictional torque in the system, obtained through a velocity observer. The estimated friction was decomposed into two components, one dominating at "zero-velocities" and one at "non-zero velocities". These components were fed through two independent adaptive estimators based on modified static models for stiction, Coulomb and viscous friction. The stability properties of the estimators were discussed, as were implementation and tuning. Experimental evaluation of the algorithm showed that, under appropriate excitation, the estimation errors were as small as $1 \%$ of the associated parameter value. This accuracy facilitates effective monitoring of equipment wear levels and, by extension, prognosis and condition-based maintenance scheduling.

\section{ACKNOWLEDGEMENTS}

The sponsorship of this project by Siemens AG is gratefully acknowledged. 


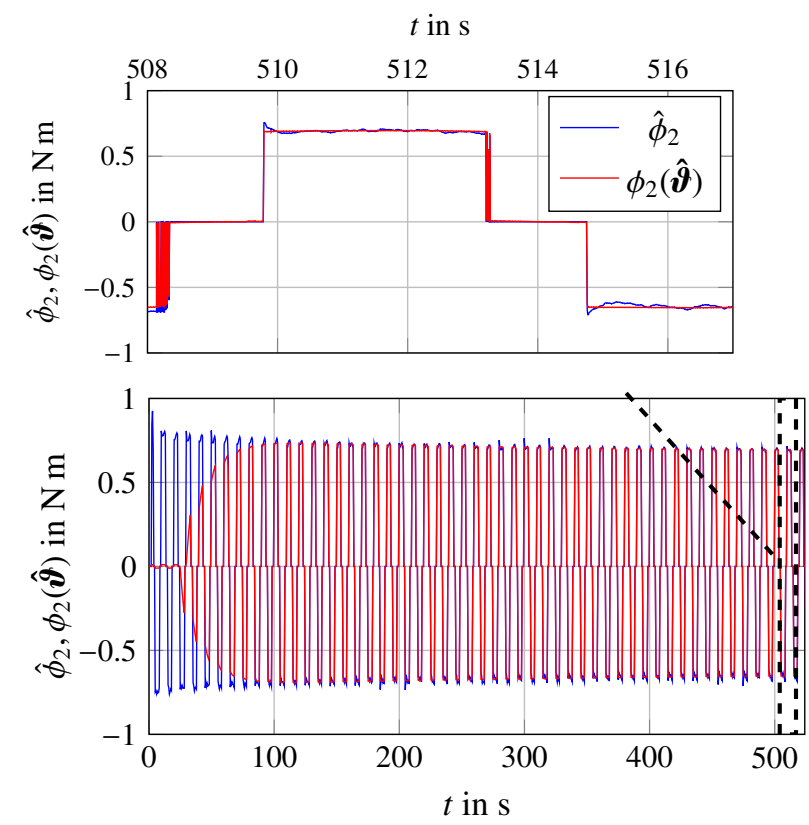

FIGURE 14 Coulomb and viscous friction $\hat{\phi}_{2}$ and its reconstructed counterpart $\phi_{2}(\hat{\boldsymbol{\vartheta}})$ based on the estimation of the Coulomb and viscous friction coefficients.

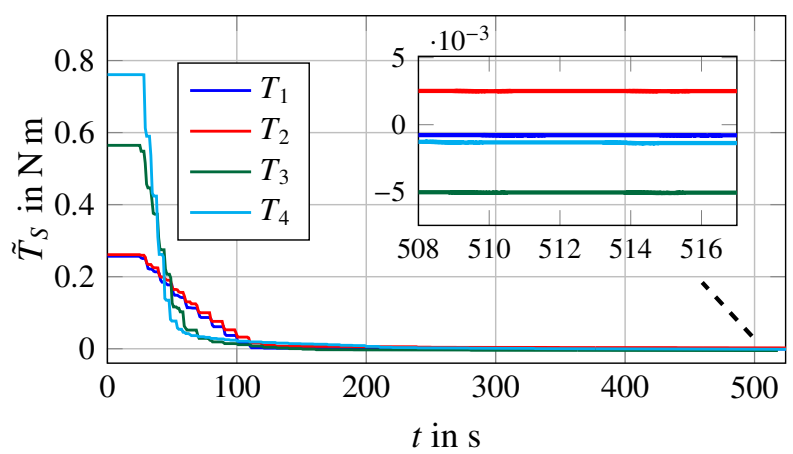

FIGURE 16 Static friction coefficient estimation error.

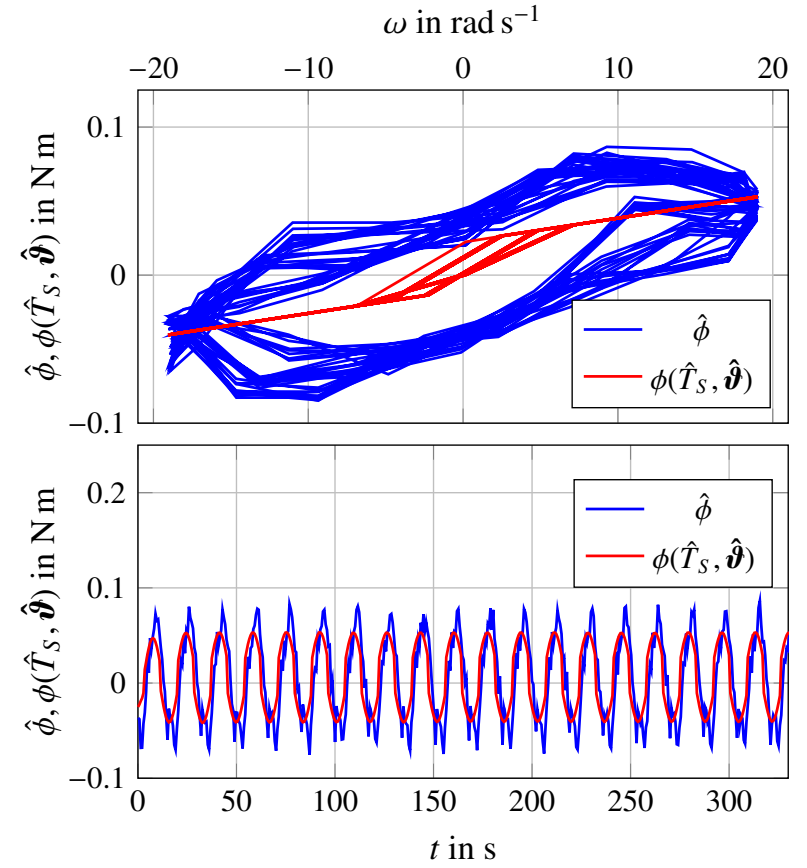

FIGURE 15 Total friction estimation and velocityfriction plot for $\theta_{r}(t)=\sin (6 \pi t)$.

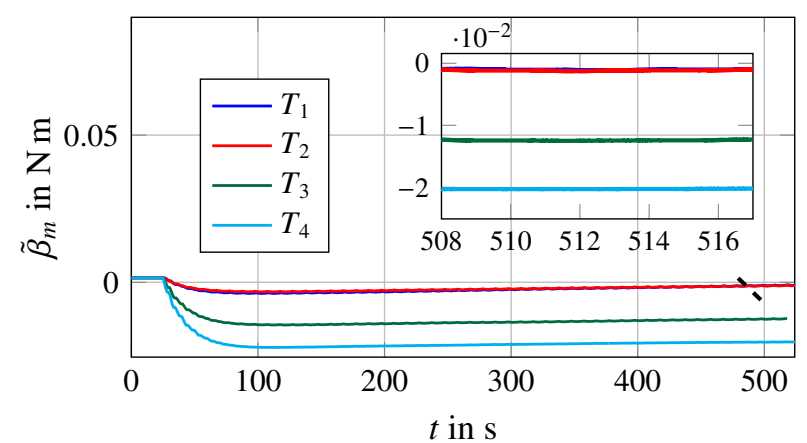

FIGURE 17 Viscous friction coefficient estimation error.

\section{APPENDIX}

\section{A THE PROJECTION OPERATOR}

Let $\Omega_{l}$ be a convex subset of the parameter space $\mathcal{P} \subset \mathbb{R}^{p}$ defined as $\Omega_{l} \triangleq\{\boldsymbol{\delta} \in \mathcal{P} \mid h(\boldsymbol{\delta}) \leq l\}$, where $l>0$ and $h: \Omega_{l} \rightarrow \mathbb{R}$ is a smooth convex function. The projection operator is defined as following ${ }^{33}$ :

$$
\operatorname{Proj}(\hat{\boldsymbol{\delta}}, \boldsymbol{\tau})= \begin{cases}\boldsymbol{\tau} & , h(\hat{\boldsymbol{\delta}})<0 \\ \boldsymbol{\tau} & , h(\hat{\boldsymbol{\delta}}) \geq 0 \& \nabla h^{T} \boldsymbol{\tau} \leq 0 \\ \boldsymbol{\tau}-\frac{\nabla h}{|\nabla h|}\left\langle\frac{\nabla h}{|\nabla h|}, \boldsymbol{\tau}\right\rangle h(\hat{\boldsymbol{\delta}}) & , h(\hat{\boldsymbol{\delta}}) \geq 0 \& \nabla h^{T} \boldsymbol{\tau}>0 .\end{cases}
$$




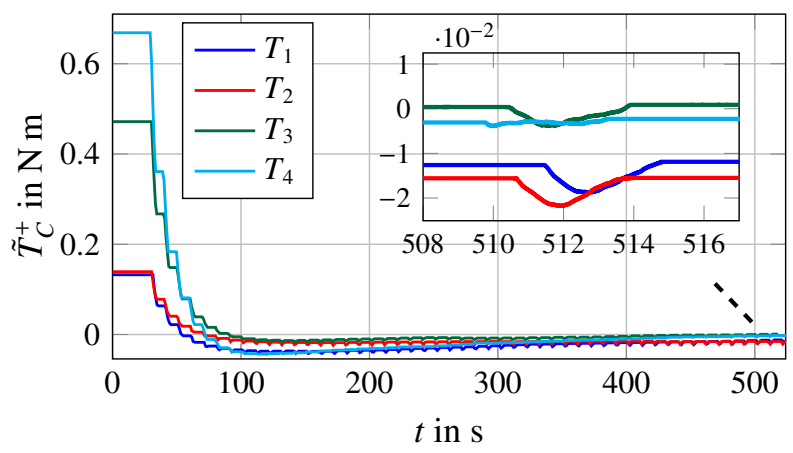

FIGURE 18 Coulomb coefficient estimation error (for positive direction).
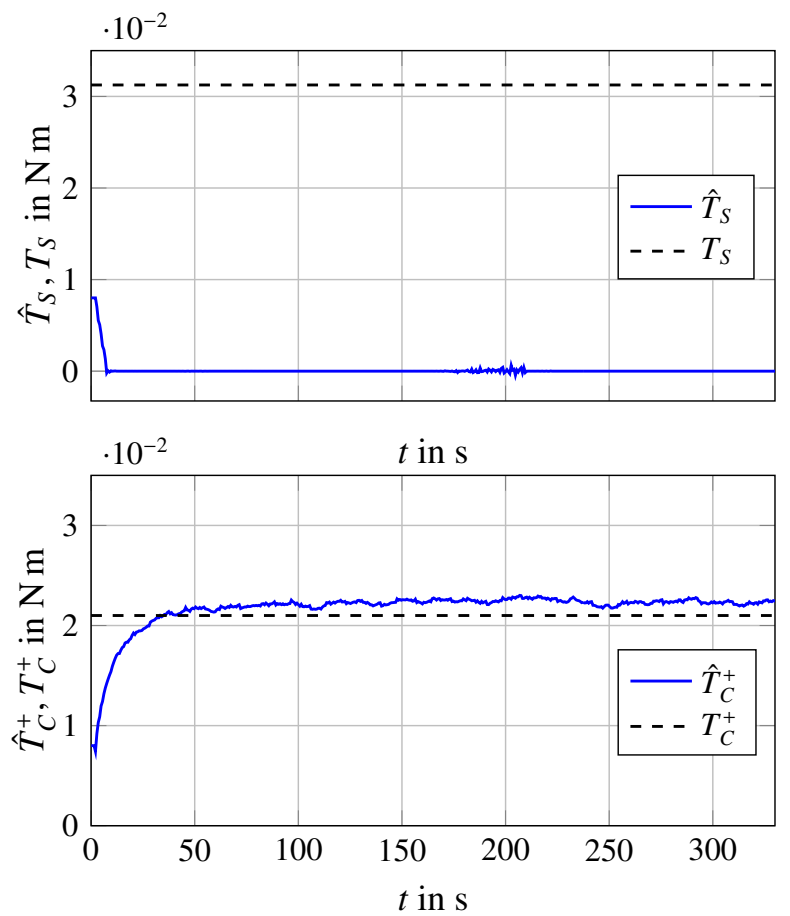

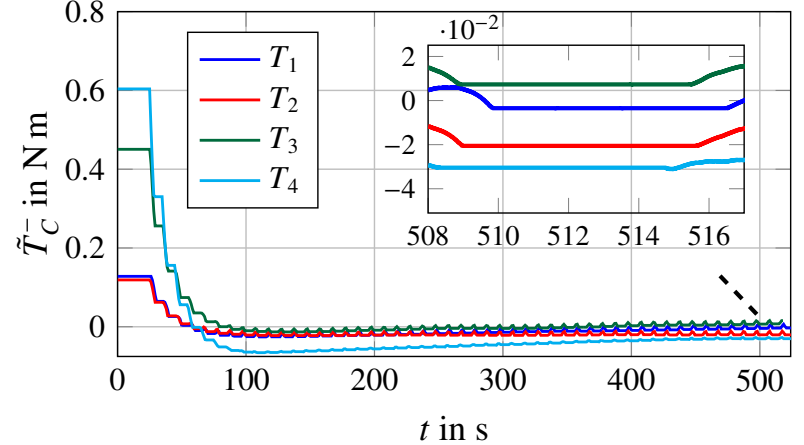

FIGURE 19 Coulomb coefficient estimation error (for negative direction).
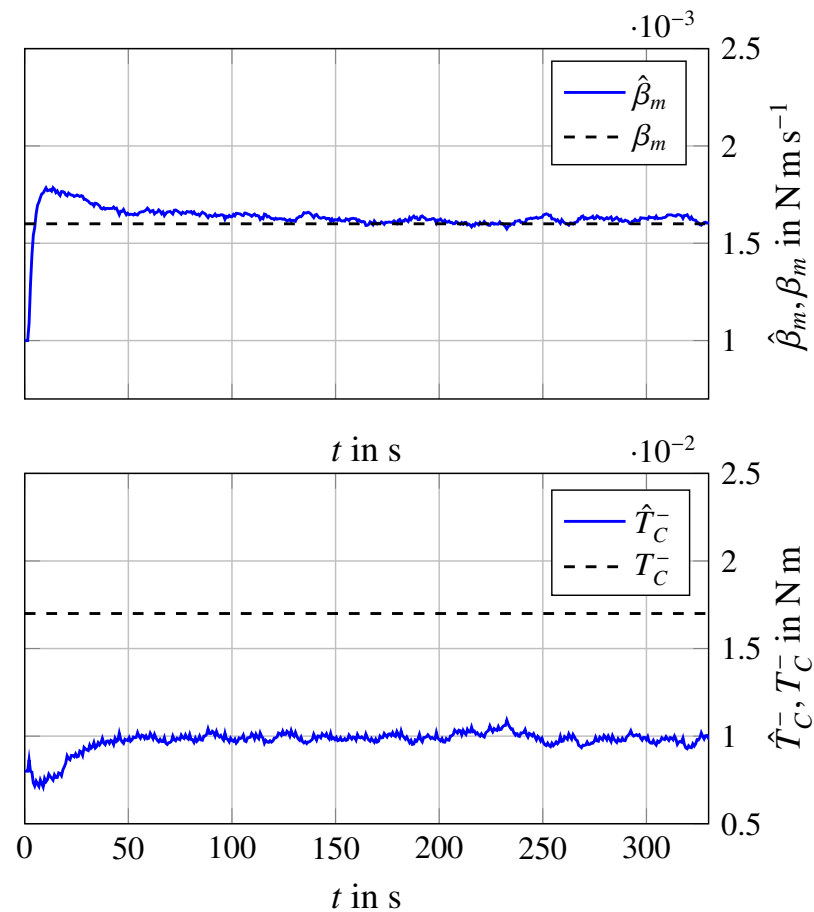

FIGURE 20 Estimation friction coefficients with $\theta_{r}(t)=\sin (6 \pi t)$. Poor excitation conditions near zero velocity lead to unreliable estimation of the static friction coefficient $T_{S}$.

In this study the convex function $h$ has been chosen according to ${ }^{33}$ :

$$
h(\hat{\boldsymbol{\delta}}) \triangleq \frac{\left(\epsilon_{\delta}+1\right) \hat{\boldsymbol{\delta}}^{T} \hat{\boldsymbol{\delta}}-\delta_{\max }^{2}}{\epsilon_{\delta} \delta_{\max }^{2}}
$$

In the above definition of $h, \delta_{\max }$ is a conservative upper bound for the parameter estimates $\hat{\delta}$ and $\epsilon_{\delta}$ is a small positive number. The operator $\langle\cdot, \cdot\rangle$ denotes the inner product, which in case of only one parameter, reduces into a real product.

\section{B PROOF OF PROPOSITION 2}

The analysis is restricted to the case where the actuating torque $u_{a}$ is not identically equal to zero and $\left|\omega_{m}\right|<\omega_{t h}$, since otherwise there is no information on the stiction phenomenon coming from the dynamics of the system or the measurements that could 

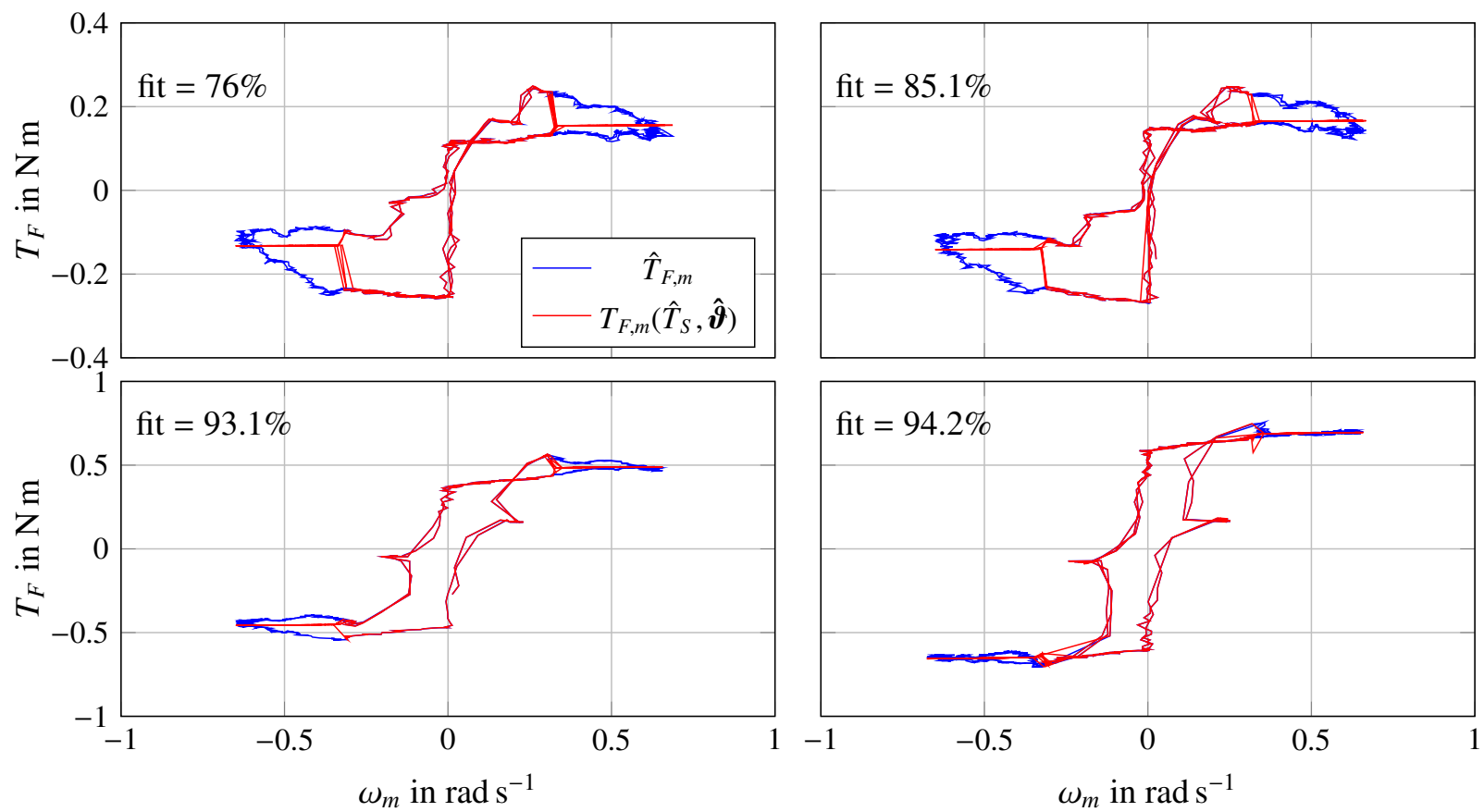

FIGURE 21 Estimated (actual) and reconstructed total friction torque (based on the estimated coefficients) as a function of the motor velocity. Tests $T_{1}, T_{2}$ at the top, $T_{3}, T_{4}$ at the bottom.

facilitate estimation of the static friction coefficient. Indeed, $\phi_{1} \equiv 0$ for $u_{a} \equiv 0$ and $\hat{\phi}_{1}=\phi_{1}=0$ for $\left|\omega_{m}\right| \geq \omega_{t h}$, which both implies that $\dot{\hat{T}}_{S}=0$. For the considered cases, the PE condition in (3.12) is only violated at a finite number of time instants where $u_{a}=0$. The proof follows the same line as in ${ }^{40, \text { Theorem } 1}{ }^{1}$. The following abbreviations are introduced:

$$
\begin{aligned}
T_{F, S} & \triangleq T_{F, S}\left(u_{a}, T_{S}^{*}\right) \\
\hat{T}_{F, S} & \triangleq T_{F, S}\left(u_{a}, \hat{T}_{S}\right) \\
\mu & \triangleq \mu\left(u_{a}, \hat{T}_{S}\right) .
\end{aligned}
$$

where $\hat{T}_{F, S}$ denotes the reconstructed stiction torque, i.e. the torque calculated by evaluating the stiction model at the estimated static friction coefficient. The term "reconstructed" will be also used for the Coulomb and viscous friction torque later on, to describe the evaluation of the associated models on the estimated Coulomb and viscous coefficients. Baring in mind that for $\left|\omega_{m}\right|<\omega_{t h}$ it holds that $s_{d}^{2}\left(\omega_{m}\right)=0$ and considering the expression in (3.8), the dynamics of the static friction coefficient estimation error reads

$$
\begin{aligned}
\dot{\tilde{T}}_{S} & =-\operatorname{Proj}\left[\hat{T}_{S}, \gamma \mu\left(u_{a}, \hat{T}_{S}\right)\left(\hat{\phi}_{1}-\phi_{1}\left(u_{a}, \hat{T}_{S}\right)\right)\right]=-\operatorname{Proj}\left[\hat{T}_{S}, \gamma \mu\left(u_{a}, \hat{T}_{S}\right)\left(\phi_{1}+\tilde{\phi}_{1}-\phi_{1}\left(u_{a}, \hat{T}_{S}\right)\right)\right] \\
& =-\operatorname{Proj}\left[\hat{T}_{S}, \gamma \mu\left(u_{a}, \hat{T}_{S}\right)\left(\tilde{\phi}_{1}+T_{F, S}-\hat{T}_{F, S}\right)\right] .
\end{aligned}
$$

Consider the continuously differentiable Lyapunov function candidate ${ }^{37,40}$

$$
V_{1}\left(t, \tilde{T}_{S}\right)=\frac{1}{2}\left(\frac{1}{\gamma}-\kappa \int_{t}^{\infty} e^{(t-\tau)} \sigma\left(u_{a}(\tau)\right) d \tau\right) \tilde{T}_{S}^{2}
$$

for which it holds

$$
\alpha_{1}\left(\left|\tilde{T}_{S}\right|\right) \leq V_{1}\left(t, \tilde{T}_{S}\right) \leq \alpha_{2}\left(\left|\tilde{T}_{S}\right|\right)
$$



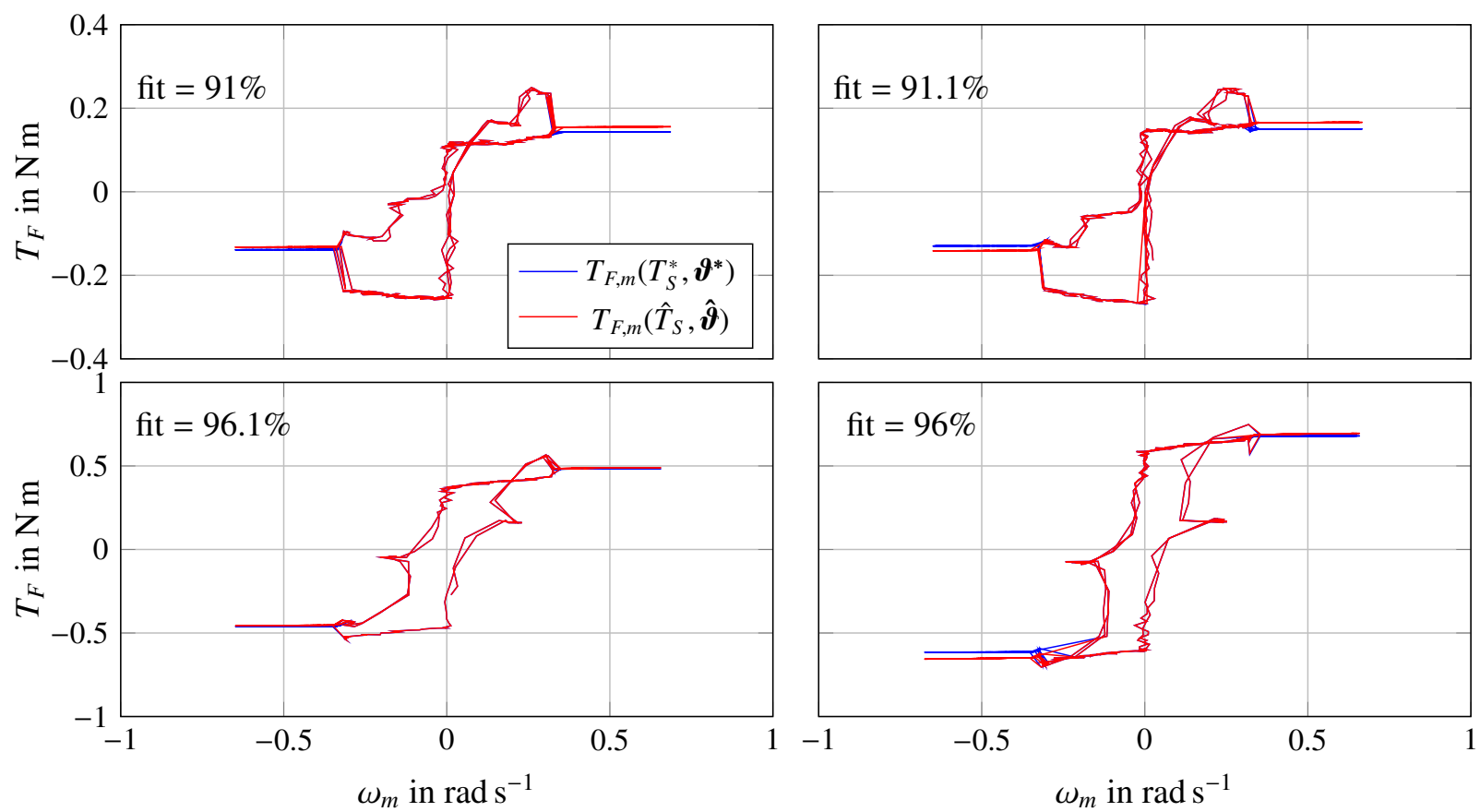

FIGURE 22 Reconstructed total friction torque as a function of the motor velocity based on the actual friction coefficients (blue) and the estimated ones (red). Tests $T_{1}, T_{2}$ at the top, $T_{3}, T_{4}$ at the bottom.

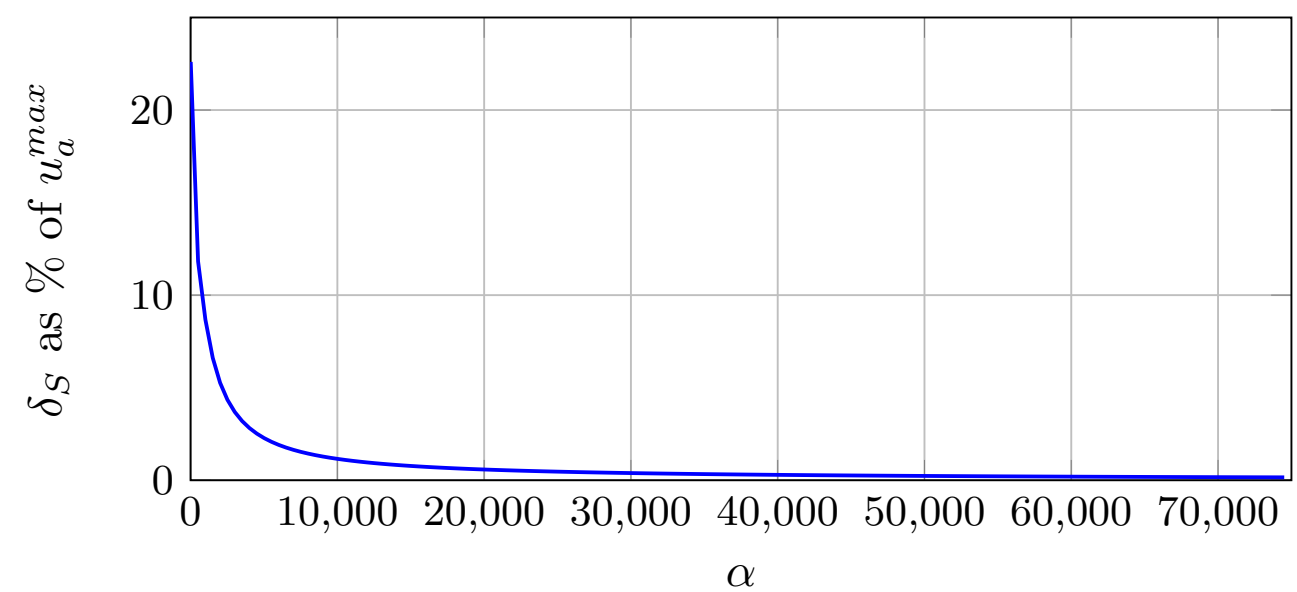

FIGURE 23 Stiction approximation error as a percentage of the actuating torque $u_{a}$ for different values of $\alpha$.

with

$$
\begin{aligned}
& \alpha_{1}\left(\left|\tilde{T}_{S}\right|\right)=\left(\frac{1}{\gamma}-\kappa \sup _{u_{a} \in \mathcal{N}} \sigma\left(u_{a}\right)\right)\left|\tilde{T}_{S}\right|^{2} \\
& \alpha_{2}\left(\left|\tilde{T}_{S}\right|\right)=\frac{1}{\gamma}\left|\tilde{T}_{S}\right|^{2}
\end{aligned}
$$

being class- $\mathcal{K}_{\infty}$ functions for $0<\kappa<\frac{1}{\gamma \sup _{\substack{1 \\ u_{a} \in \mathcal{N}}} \sigma\left(u_{a}\right)}$ inside $\mathcal{N} \triangleq \mathcal{V} \times \mathcal{D}-\left\{u_{a} \in \mathcal{V} \times \mathcal{D} \mid \sigma\left(u_{a}\right)=0\right\}$ and where $\kappa$ will be defined later. The function $V$ is positive definite since it is bounded from both sides by class- $\mathcal{K}_{\infty}$ functions. Its time-derivative along the 
trajectories of the estimation error is given by

$$
\begin{aligned}
\dot{V}_{1}\left(t, \tilde{T}_{S}\right) & =\frac{\partial V}{\partial t}\left(t, \tilde{T}_{S}\right)+\frac{\partial V}{\partial \tilde{T}_{S}}\left(t, \tilde{T}_{S}\right) \dot{\tilde{T}}_{S}=\frac{1}{2} \tilde{T}_{S}^{2} \frac{\partial}{\partial t}\left[-\kappa \int_{t}^{\infty} e^{(t-\tau)} \sigma\left(u_{a}(\tau)\right) d \tau\right]+\tilde{T}_{S}\left(\frac{1}{\gamma}-\kappa \int_{t}^{\infty} e^{(t-\tau)} \sigma\left(u_{a}(\tau)\right) d \tau\right) \dot{\tilde{T}}_{S} \leq \\
& \leq \frac{\kappa}{2}\left(\sigma\left(u_{a}\right)-e^{-T} \int_{t}^{t+T} \sigma\left(u_{a}(\tau)\right) d \tau\right) \tilde{T}_{S}^{2}-\tilde{T}_{S}\left(\frac{1}{\gamma}-\kappa \int_{t}^{\infty} e^{(t-\tau)} \sigma\left(u_{a}(\tau)\right) d \tau\right) \gamma \mu\left(u_{a}, \hat{T}_{S}\right)\left(T_{F, S}-\hat{T}_{F, S}\right) \\
& -\tilde{T}_{S}\left(\frac{1}{\gamma}-\kappa \int_{t}^{\infty} e^{(t-\tau)} \sigma\left(u_{a}(\tau)\right) d \tau\right) \gamma \mu\left(u_{a}, \hat{T}_{S}\right) \tilde{\phi}_{1} .
\end{aligned}
$$

where the properties ${ }^{43}$, Lemma E. 1

$$
\begin{gathered}
-\tilde{T}_{S} \operatorname{Proj}\left[\hat{T}_{S}, \gamma \mu\left(u_{a}, \hat{T}_{S}\right)\left(\tilde{\phi}_{1}+T_{F, S}-\hat{T}_{F, S}\right)\right] \leq-\gamma \tilde{T}_{S} \mu\left(u_{a}, \hat{T}_{S}\right)\left(\tilde{\phi}_{1}+T_{F, S}-\hat{T}_{F, S}\right) \\
\text { and } \int_{0}^{\infty} e^{(t-\tau)} \sigma(x(\tau)) d \tau \geq e^{-T} \int_{t}^{t+T} \sigma(x(\tau)) d \tau, T>0
\end{gathered}
$$

where used. Applying the equality ${ }^{44}$, Theorem 11.1

$$
T_{F, S}(u_{a}, \underbrace{\hat{T}_{S}+\tilde{T}_{S}}_{T_{S}^{*}})-T_{F, S}\left(u_{a}, \hat{T}_{S}\right)=\int_{0}^{1} \frac{\partial T_{F, S}}{\partial \hat{T}_{S}}\left(u_{a}, \hat{T}_{S}+p \tilde{T}_{S}\right) \tilde{T}_{S} d p,
$$

as well as (3.12) and after straightforward calculations, inequality (B5) leads to

$$
\begin{aligned}
\dot{V}_{1}\left(t, \tilde{T}_{S}\right) & \leq \frac{\kappa}{2}\left(\sigma\left(u_{a}\right)-e^{-T} \varepsilon\right) \tilde{T}_{S}^{2}-\tilde{T}_{S} \int_{0}^{1} \mu\left(u_{a}, \hat{T}_{S}\right) \frac{\partial T_{F, S}}{\partial \hat{T}_{S}}\left(u_{a}, \hat{T}_{S}+p \tilde{T}_{S}\right) d p \\
& +\left|\tilde{T}_{S}\right| \kappa \gamma\left|\mu\left(u_{a}, \hat{T}_{S}\right)\right|\left|\int_{t}^{\infty} e^{(t-\tau)} \sigma\left(u_{a}(\tau)\right) d \tau\right|\left|T_{F, S}-\hat{T}_{F, S}\right|-\tilde{T}_{S}\left(\frac{1}{\gamma}-\kappa \int_{t}^{\infty} e^{(t-\tau)} \sigma\left(u_{a}(\tau)\right) d \tau\right) \gamma \mu\left(u_{a}, \hat{T}_{S}\right) \tilde{\phi}_{1} .
\end{aligned}
$$

Introducing the upper bounds $M, \Sigma$ of the functions $\mu\left(u_{a}, \hat{T}_{S}\right), \sigma\left(u_{a}\right)$, respectively and using the properties (3.10),(3.11) gives

$$
\begin{aligned}
& \dot{V}_{1}\left(t, \tilde{T}_{S}\right) \leq-\left(1-\frac{\kappa}{2}\right) \sigma\left(u_{a}\right) \tilde{T}_{S}^{2}-\frac{\kappa}{2} \varepsilon e^{-T} \tilde{T}_{S}^{2}-\tilde{T}_{S}\left(\frac{1}{\gamma}-\kappa \int_{t}^{\infty} e^{(t-\tau)} \sigma\left(u_{a}(\tau)\right) d \tau\right) \gamma \mu\left(u_{a}, \hat{T}_{S}\right) \tilde{\phi}_{1}+\kappa \gamma M \Sigma L \sqrt{\sigma\left(u_{a}\right)}= \\
& =-\left|\tilde{T}_{S}\right|^{2}\left[\begin{array}{c}
1 \\
\sqrt{\sigma\left(u_{a}\right)}
\end{array}\right]^{T} \underbrace{\left[\begin{array}{cc}
\frac{\kappa}{2} \varepsilon e^{-T} r^{-\frac{\kappa}{2} \gamma \cdot M \cdot \Sigma \cdot L} \\
-\frac{\kappa}{2} \gamma \cdot M \cdot \Sigma \cdot L & 1-\frac{\kappa}{2}
\end{array}\right]}_{Q}\left[\begin{array}{c}
1 \\
\sqrt{\sigma\left(u_{a}\right)}
\end{array}\right]-\tilde{T}_{S}\left(\frac{1}{\gamma}-\kappa \int_{t}^{\infty} e^{(t-\tau)} \sigma\left(u_{a}(\tau)\right) d \tau\right) \gamma \mu\left(u_{a}, \hat{T}_{S}\right) \tilde{\phi}_{1} .
\end{aligned}
$$

Choosing

$$
\kappa<\frac{2}{1+\frac{e^{T}}{\varepsilon} M^{2} \Sigma^{2} \gamma^{2} L^{2}}
$$

renders $Q$ positive definite and denoting its minimum eigenvalue with $\lambda$ finally leads to

$$
\dot{V}_{1}\left(t, \tilde{T}_{S}\right) \leq-\lambda\left|\tilde{T}_{S}\right|^{2}-\tilde{T}_{S}\left(\frac{1}{\gamma}-\kappa \int_{t}^{\infty} e^{(t-\tau)} \sigma\left(u_{a}(\tau)\right) d \tau\right) \gamma \mu\left(u_{a}, \hat{T}_{S}\right) \tilde{\phi}_{1} .
$$

Three cases are concerned: 
Case 1: If $\hat{\phi}_{1}=\phi_{1}\left(u_{a}, T_{s}\right)$, then $\tilde{\phi}_{1}=0$. In this case (B8) implies that $\dot{V}$ is negative definite and since

$$
\left|\frac{\partial V_{1}}{\partial \tilde{T}_{S}}\right|=\left|\tilde{T}_{S}\left(\frac{1}{\gamma}-\kappa \int_{t}^{\infty} e^{(t-\tau)} \sigma\left(u_{a}(\tau)\right) d \tau\right)\right| \leq\left|\frac{1}{\gamma}-\kappa e^{-T} \Sigma T\right|\left|\tilde{T}_{S}\right|, T>0
$$

which implies that $\frac{\partial V}{\partial \tilde{\delta}}$ is Uniformly Bounded (UB), the equilibrium point $\tilde{T}_{S}^{*}=0$ is $\operatorname{ULES}^{45}$.

Case 2: The dynamics of the stiction coefficient estimation error in (B1) expresses the cascaded interconnection of the two following systems:

- The unperturbed estimation error system $\left(\Sigma_{1}\right)$

$$
\dot{\tilde{T}}_{S}=-\operatorname{Proj}\left[\hat{T}_{S}, \gamma \mu\left(u_{a}, \hat{T}_{S}\right)\left(T_{F, S}-\hat{T}_{F, S}\right)\right]
$$

for which the origin $\tilde{T}_{S}^{*}=0$ is a ULES equilibrium point.

- The dynamical system $\left(\Sigma_{2}\right)$, whose output is the stiction estimation error $\tilde{\phi}_{1}$ (see Figure B1).

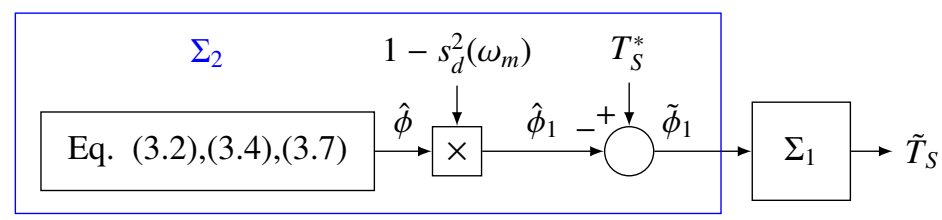

FIGURE B1 Cascaded interconnection of systems $\left(\Sigma_{1}\right)$ and $\left(\Sigma_{2}\right)$.

If $\tilde{\phi}_{1}^{*}=0$, is UGAS, then under the condition that $\left(\Sigma_{1}\right)$ is Input-to-State Stable (ISS) with respect to the perturbation $\tilde{\phi}_{1}$, the origin $\tilde{T}_{S}^{*}=0$ is a UGAS equilibrium point of (B1) ${ }^{46, \text { Lemma } 1}$. Indeed (B8) implies

$$
\dot{V}_{1}\left(t, \tilde{T}_{S}\right) \leq\left|\tilde{T}_{S}\right|\left[\left(1-\kappa \gamma e^{-T} \varsigma \cdot T\right) M\left|\tilde{\phi}_{1}\right|-q \lambda\left|\tilde{T}_{S}\right|\right]-(1-q) \lambda\left|\tilde{T}_{S}\right|^{2} \leq-(1-q) \lambda\left|\tilde{T}_{S}\right|^{2}
$$

for all $\tilde{T}_{S} \in S$ with

$$
\left|\tilde{T}_{S}\right| \geq \underbrace{\frac{\left(1-\kappa \gamma e^{-T} \varsigma \cdot T\right) M}{\lambda q}}_{P}\left|\tilde{\phi}_{1}\right|=P\left|\tilde{\phi}_{1}\right|
$$

with $0<q<1$ and $\varsigma \geq 0$ being a lower bound for $\sigma\left(u_{a}\right)$. Then according to definition $4.7 \mathrm{in}^{45},\left(\Sigma_{1}\right)$ is ISS with respect to $\tilde{\phi}_{1}$.

Case 3: The ISS property of the system $\left(\Sigma_{1}\right)$ implies the uniform global boundedness of the cascaded interconnection and specifically it holds that:

$$
\left|\tilde{T}_{S}(t)\right| \leq \beta_{1}\left(\left|\tilde{T}_{S}\left(t_{0}\right)\right|, t-t_{0}\right)+\beta_{2}\left(\sup _{0 \leq \tau \leq t} \tilde{\phi}_{1}(\tau)\right), \forall\left|\tilde{T}_{S}\right| \geq P\left|\tilde{\phi}_{1}\right|
$$

where $\beta_{1}, \beta_{2}$ are class- $\mathcal{K L}$ and class- $\mathcal{K}$ functions, respectively. More specifically, if $\Phi_{1}$ is an upper bound of $\left|\tilde{\phi}_{1}\right|$, then $\beta_{2}$ can be explicitly found by 45 , Th. 4.19 :

$$
\beta_{2}\left(\sup _{0 \leq \tau \leq t} \tilde{\phi}_{1}(\tau)\right)=\alpha_{1}^{-1} \circ \alpha_{2} \circ\left(P \Phi_{1}\right)=\frac{P}{\sqrt{1-\kappa \sup _{u_{a} \in \mathcal{N}} \sigma\left(u_{a}\right) \cdot \gamma}} \Phi_{1}=c \Phi_{1}
$$

where $\alpha_{1}, \alpha_{2}$ were defined in (B3),(B4).

Since also $\beta_{1} \in \mathcal{K} \mathcal{L}$, taking the limit of (B11) for $t \rightarrow \infty$ yields:

$$
\lim _{t \rightarrow \infty}\left|\tilde{T}_{S}(t)\right| \leq \lim _{t \rightarrow \infty}\left(\beta\left(\left|\tilde{T}_{S}\left(t_{0}\right)\right|, t-t_{0}\right)+c \Phi_{1}\right)=c \Phi_{1}
$$

which completes the proof. 


\section{References}

1. Rebelein C, Zaeh M. Friction in feed drives of machine tools: investigation, modeling and validation. Production Engineering 2016: 1-11.

2. Ruderman M, Iwasaki M. Analysis of linear feedback position control in presence of presliding friction. Ieej Journal of Industry Applications 2016; 5(2): 61-68. doi: 10.1541/ieejjia.5.61

3. Gross H, Hamann J, Wiegärtner G. Electrical feed drives in automation: basics, computation, dimensioning. Publicis MCD Corporate Pub. . 2001.

4. Straffelini G. Friction and Wear: Methodologies for Design and Control (Springer Tracts in Mechanical Engineering). Springer. 1 ed. 2015.

5. Armstrong-Hélouvry B, Dupont P, Wit CCD. A survey of models, analysis tools and compensation methods for the control of machines with friction. Automatica 1994; 30(7): 1083 - 1138. doi: 10.1016/0005-1098(94)90209-7

6. Johanastrom K, Canudas-De-Wit C. Revisiting the LuGre friction model. IEEE control Systems 2008; 28(6): 101-114.

7. Al-Bender F, Swevers J. Characterization of Friction Force Dynamics. Ieee Control Systems 2008; 28(6): 64-81. doi: 10.1109/MCS.2008.929279

8. Boegli M, De Laet T, De Schutter J, Swevers J. A Smoothed GMS friction model for Moving Horizon friction state and parameter estimation. In: IEEE. ; 2012: 1-6.

9. Swevers J, Al-Bender F, Ganseman CG, Prajogo T. An integrated friction model structure with improved presliding behavior for accurate friction compensation. Ieee Transactions on Automatic Control 2000; 45(4): 675-686. doi: 10.1109/9.847103

10. Lampaert V, Swevers J, Al-Bender F. Modification of the Leuven integrated friction model structure. IEEE transactions on Automatic Control 2002; 47(4): 683-687.

11. Ferretti G, Magnani G, Rocco P. An integral friction model. In: . 2. IEEE. ; 2004: 1809-1813.

12. Ferretti G, Magnani G, Rocco P. Single and multistate integral friction models. IEEE Transactions on Automatic Control 2004; 49(12): 2292-2297.

13. Ruderman M, Bertram T. Two-state dynamic friction model with elasto-plasticity. Mechanical Systems and Signal Processing 2013; 39(1): 316-332.

14. Indri M, Lazzero I, Antoniazza A, Bottero AM. Friction modeling and identification for industrial manipulators. In: IEEE. ; 2013: 1-8.

15. Wan Y, Dodd T, Wong C, Harrison R, Worden K. Kernel based modelling of friction dynamics. Mechanical Systems and Signal Processing 2008; 22(1): 66-80.

16. De Wit CC, Lischinsky P. Adaptive friction compensation with partially known dynamic friction model. International journal of adaptive control and signal processing 1997; 11(1): 65-80.

17. Hensen RH, Molengraft v. dMJ, Steinbuch M. Frequency domain identification of dynamic friction model parameters. IEEE Transactions on Control Systems Technology 2002; 10(2): 191-196.

18. Ravanbod-Shirazi L, Besançon-Voda A. Friction identification using the Karnopp model, applied to an electropneumatic actuator. Proceedings of the Institution of Mechanical Engineers, Part I: Journal of Systems and Control Engineering 2003; 217(2): 123-138.

19. Márton L, Fodor S, Sepehri N. A practical method for friction identification in hydraulic actuators. Mechatronics 2011; 21(1): 350-356.

20. Stevanović N, Green PL, Worden K, Kirkegaard PH. Friction estimation in wind turbine blade bearings. Structural Control and Health Monitoring 2016; 23(1): 103-122. 
21. Kim MS, Chung SC. Friction identification of ball-screw driven servomechanisms through the limit cycle analysis. Mechatronics 2006; 16(2): 131-140.

22. Yoon JY, Trumper DL. Friction modeling, identification, and compensation based on friction hysteresis and Dahl resonance. Mechatronics 2014; 24(6): 734-741.

23. Wenjing Z. Parameter identification of LuGre friction model in servo system based on improved particle swarm optimization algorithm. In: IEEE. ; 2007: 135-139.

24. Rizos DD, Fassois SD. Friction identification based upon the LuGre and Maxwell slip models. IEEE Transactions on Control Systems Technology 2009; 17(1): 153-160.

25. Ruderman M. Discrete-time series identification of sliding dynamic friction in industrial robotic joints. In: IEEE. ; 2013: $5809-5814$.

26. Altpeter F, Grunenberg M, Myszkorowski P, Longchamp R. Auto-tuning of feedforward friction compensation based on the gradient method. Proceedings of the American Control Conference 2000; 4: 2600-2604. doi: 10.1109/ACC.2000.878677

27. Amthor A, Zschaeck S, Ament C. High precision position control using an adaptive friction compensation approach. Ieee Transactions on Automatic Control 2010; 55(1): 5350440, 274-278. doi: 10.1109/TAC.2009.2036307

28. Liu YF, Li J, Zhang ZM, Hu XH, Zhang WJ. Experimental comparison of five friction models on the same test-bed of the micro stick-slip motion system. Mechanical Sciences 2015; 6(1): 15-28.

29. Pennestrì E, Rossi V, Salvini P, Valentini PP. Review and comparison of dry friction force models. Nonlinear Dynamics 2016; 83(4): 1785-1801. doi: 10.1007/s11071-015-2485-3

30. Egeland O, Gravdahl JT. Modeling and simulation for automatic control. 76. Marine Cybernetics Trondheim, Norway . 2002.

31. Karnopp D. Computer Simulation of Stick-Slip Friction in Mechanical Dynamic Systems. Journal of Dynamic Systems, Measurement, and Control 1985; 107(1): 100. doi: 10.1115/1.3140698

32. Jelali M, Huang B. Detection and diagnosis of stiction in control loops: state of the art and advanced methods. Springer Science \& Business Media . 2009.

33. Hovakimyan N, Cao C. L1 Adaptive Control Theory: Guaranteed Robustness with Fast Adaptation (Advances in Design and Control). Society for Industrial and Applied Mathematics,U.S. . 2010.

34. Cao C, Annaswamy AM, Kojic A. Parameter Convergence in Nonlinearly Parameterized Systems. IEEE Transactions on Automatic Control 2003; 48(3): 1-16.

35. Tyukin IY, Prokhorov DV, Leeuwen vC. Adaptation and parameter estimation in systems with unstable target dynamics and nonlinear parametrization. IEEE Transactions on Automatic Control 2007; 52(9): 1543-1559. doi: 10.1109/TAC.2007.904448

36. Tyukin IY, Rogachev P, Nijmeijer H. Adaptive observers for nonlinearly parameterized systems subjected to parametric constraints. IFAC Proceedings Volumes (IFAC-PapersOnline) 2014; 19: 10869-10874. doi: 10.3182/20140824-6-ZA1003.00807

37. Grip HF, Johansen TA, Imsland L, Kaasa Go. Parameter estimation and compensation in systems with nonlinearly parameterized perturbations. Automatica 2010; 46(1): 19-28. doi: 10.1016/j.automatica.2009.10.013

38. Grip HF, Saberi A, Johansen TA. Estimation of states and parameters for linear systems with nonlinearly parameterized perturbations. Systems and Control Letters 2011; 60(9): 771-777. doi: 10.1016/j.sysconle.2011.03.012

39. Grip HF, Saberi A. High-gain observer design for multi-output systems: Transformation to a canonical form by dynamic output shaping. International Journal of Robust and Nonlinear Control 2014; 24(6): 1016-1042. doi: 10.1002/rnc.2900 
40. Papageorgiou D, Blanke M, Niemann HH, Richter JH. Robust Backlash Estimation for Industrial Drive-Train SystemsTheory and Validation. IEEE Transactions on Control Systems Technology 2018(99): 1-15.

41. Dyratok H. Huco Vari-tork adjustable-friction clutches.; 2017. http://www.huco.com/products.asp?p=true\&cat=155 [Accessed: January 27].

42. Siemens . SIMOTICS IFT7 servomotors.; $2017 . \quad \mathrm{http} / / \mathrm{w} 3$. siemens.com/mcms/mc-solutions/en/motors/ motion-control-motors/simotics-s-servomotors/simotics-s-1ft7/pages/simotics-s-1ft7.aspx [Accessed: October 03].

43. Krstic M, Kanellakopoulos I, Kokotovic PV. Nonlinear and Adaptive Control Design. Wiley-Interscience. 1 ed. 1995.

44. Wright S, Nocedal J. Numerical optimization. Springer Science 1999; 35: 67-68.

45. Khalil H. Nonlinear Systems. Prentice Hall . 2002.

46. Loria A, Panteley E. Cascaded nonlinear time-varying systems: Analysis and design. Lecture Notes in Control and Information Sciences 2005; 311: 23-64. 


\section{AUTHOR BIOGRAPHY}

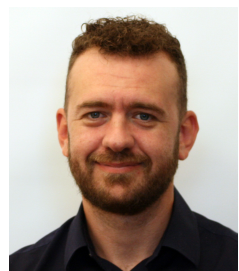

Dimitrios Papageorgiou received his M.Sc. and Ph.D. degrees from the Technical University of Denmark (DTU) in 2014 and 2017, respectively. He is currently a post-doctoral researcher at the Automation and Control group of the electrical engineering department in DTU. His research interests focus in nonlinear and adaptive control and estimation for electromechanical systems, fault-diagnosis and system monitoring, as well as control for marine applications.

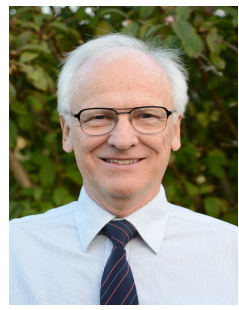

Mogens Blanke (M 1974, SM 1985) received the MScEE degree in 1974 and the PhD degree in 1982 from the Technical University of Denmark, DTU. He was Systems Analyst with the European Space Agency 197576, with DTU 1977-84, was Head of Division at Lyngs $\varnothing$ Marine 1985-89, Professor at Aalborg University 1990-99. Since 2000, he has been Professor in Automation and Control at DTU and also Adjunct Professor at the Norwegian University of Science and Technology 2005-17. Areas of special focus include diagnosis, prognosis and fault tolerant control. Prof. Blanke has held various positions in the International Federation of Automatic Control, including Chair of the TC on Marine Systems, CC Chair and Member of Council. He is a member of the IFAC SAFEPROCESS and Marine Systems TCs. He was Technical Editor for IEEE Transactions of Aerospace and Electronic Systems (2006-2016) and is currently Associate Editor for Control Engineering Practice and Deputy Editor for Ocean Engineering.

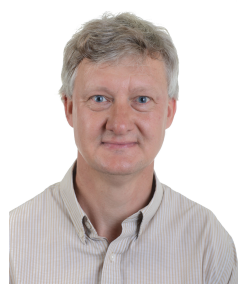

Hans Henrik Niemann was born in Denmark in 1961. He received the M.Sc degree in mechanical engineering in 1986 and the Ph.D. degree in 1988 from Technical University of Denmark. From 1988 to 1994 he has a research position and from 1994 he has been Ass. Professor in control engineering at Technical University of Denmark. His research interests are: Optimal and robust control, fault detection and isolation, active fault diagnosis, fault tolerant control, controller architecture for controller switching and fault tolerant control, system and performance monitoring, controller anti-windup. He is first author of more than 80 journal and conferences papers.

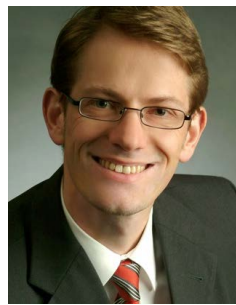

Jan H. Richter received the diploma in electrical engineering and the PhD in control theory from TU Hamburg-Harburg and Ruhr-Universität Bochum in 2004 and 2009, respectively. He is currently an expert in control, project manager, and researcher at Siemens AG, Digital Factory Division. His areas of interest include the toolchain for model-based systems engineering, model-based controller design, verification and validation, and fault-tolerant control in embedded and factory automation applications. 